\title{
Ultradiscrete sine-Gordon Equation over Symmetrized Max-Plus Algebra, and Noncommutative Discrete and Ultradiscrete sine-Gordon Equations
}

Kenichi KONDO

5-13-12-207 Matsubara, Setagaya-ku, Tokyo 156-0043, Japan

E-mail: incidence_algebra@poset.jp

Received January 08, 2013, in final form October 31, 2013; Published online November 12, 2013

http://dx.doi.org/10.3842/SIGMA.2013.068

\begin{abstract}
Ultradiscretization with negative values is a long-standing problem and several attempts have been made to solve it. Among others, we focus on the symmetrized max-plus algebra, with which we ultradiscretize the discrete sine-Gordon equation. Another ultradiscretization of the discrete sine-Gordon equation has already been proposed by previous studies, but the equation and the solutions obtained here are considered to directly correspond to the discrete counterpart. We also propose a noncommutative discrete analogue of the sine-Gordon equation, reveal its relations to other integrable systems including the noncommutative discrete KP equation, and construct multisoliton solutions by a repeated application of Darboux transformations. Moreover, we derive a noncommutative ultradiscrete analogue of the sine-Gordon equation and its 1-soliton and 2-soliton solutions, using the symmetrized max-plus algebra. As a result, we have a complete set of commutative and noncommutative versions of continuous, discrete, and ultradiscrete sine-Gordon equations.
\end{abstract}

Key words: ultradiscrete sine-Gordon equation; symmetrized max-plus algebra; noncommutative discrete sine-Gordon equation; noncommutative ultradiscrete sine-Gordon equation

2010 Mathematics Subject Classification: 37K10; 39A12

\section{Introduction}

\subsection{Ultradiscretization and its problem}

Ultradiscrete integrable systems are integrable systems where independent variables take values in $\mathbb{Z}$, and dependent variables in the max-plus algebra $\mathbb{R}_{\max }=\mathbb{R} \cup\{-\infty\}$. Among them is the famous box-ball system [21], represented by the equation

$$
U_{n}^{t+1}=\min \left[1-U_{n}^{t}, \sum_{k=-\infty}^{n-1} U_{k}^{t}-\sum_{k=-\infty}^{n-1} U_{k}^{t+1}\right] .
$$

Defining $S_{n}^{t}=\sum_{k=n}^{\infty} \sum_{l=-\infty}^{t} U_{k}^{l}$, we obtain

$$
S_{n+1}^{t+1}+S_{n}^{t-1}=\max \left[S_{n+1}^{t-1}+S_{n}^{t+1}-1, S_{n}^{t}+S_{n+1}^{t}\right]
$$

which is ultradiscretization of the discrete $\mathrm{KdV}$ equation

$$
(1+\delta) \sigma_{n+1}^{t+1} \sigma_{n}^{t-1}=\delta \sigma_{n+1}^{t-1} \sigma_{n}^{t+1}+\sigma_{n}^{t} \sigma_{n+1}^{t}
$$


Ultradiscretization [22] is a systematic procedure to obtain ultradiscrete systems from discrete systems. The fundamental formula of the procedure is

$$
\lim _{\epsilon \rightarrow+0} \epsilon \log \left(e^{A / \epsilon}+e^{B / \epsilon}\right)=\max [A, B], \quad \lim _{\epsilon \rightarrow+0} \epsilon \log \left(e^{A / s} \cdot e^{B / s}\right)=A+B .
$$

This may be understood as a transformation of addition into max operation and of multiplication into addition. Setting $\delta=e^{-1 / \epsilon}$ in (1.2) and applying ultradiscretization, we obtain (1.1).

The problem is, however, that ultradiscretization cannot be applied to subtraction, which is of course necessary in many discrete integrable systems. The reason is as follows. If one wants to define an ultradiscrete version of subtraction, it is natural to solve the linear equation

$$
\max [x, a]=b
$$

for $x \in \mathbb{R}_{\max }$. This has no solution when $a>b$, and therefore subtraction cannot be defined in general.

Several attempts $[8,11,15,18,19,23]$ have been made to solve this problem. We focus on the symmetrized max-plus algebra $[1,2]$, denoted by $u \mathbb{R}$ in this paper. This algebra is an extension of $\mathbb{R}_{\max }$ and looks natural in the sense it traces the construction of $\mathbb{Z}$ from $\mathbb{N}^{2}$. Linear algebra over $u \mathbb{R}$ is also possible, and ultradiscretization with $u \mathbb{R}$ is presented in [4]. These theories of $u \mathbb{R}$ are mainly developed in the field of discrete event systems and seems little known to the field of integrable systems.

\subsection{Contents of the paper}

The discrete sine-Gordon equation $[3,7]$

$$
\begin{aligned}
& (1-\delta) \tau_{l}^{m} \tau_{l+1}^{m+1}=\tau_{l+1}^{m} \tau_{l}^{m+1}-\delta \sigma_{l+1}^{m} \sigma_{l}^{m+1}, \\
& (1-\delta) \sigma_{l}^{m} \sigma_{l+1}^{m+1}=\sigma_{l+1}^{m} \sigma_{l}^{m+1}-\delta \tau_{l+1}^{m} \tau_{l}^{m+1}
\end{aligned}
$$

has not been ultradiscretized until recent years because soliton solutions include subtraction or even complex numbers. The first attempt is made by Isojima et al. $[9,10]$ where a $\tau$-only trilinear equation is exploited to exclude subtraction. Here we propose another method to ultradiscretize the sine-Gordon equation which utilizes $u \mathbb{R}$. The equation and the solutions are ultradiscretized keeping subtraction and complex numbers in a highly direct fashion.

Noncommutative integrable systems have been drawing more interest in the last two decades. It is difficult to point out the first appearance of such systems, but the noncommutative KdV equation is already mentioned in [13]. The first discrete noncommutative integrable system is probably the noncommutative discrete $\mathrm{KP}$ equation [12, 17]. Along this line, we propose a noncommutative discrete analogue of the sine-Gordon equation, explore relations to other integrable systems, and construct multisoliton solutions by the Darboux transformation. Moreover, we also propose a noncommutative ultradiscrete analogue of the sine-Gordon equation and explicitly derive 1 -soliton and 2 -soliton solutions by ultradiscretization with uR. As a result, we have a complete set of commutative and noncommutative versions of continuous, discrete, and ultradiscrete sine-Gordon equations.

The rest of the paper is organized as follows. In Section 2.1, the discrete sine-Gordon equation and 1-soliton and 2-soliton solutions are reviewed. Special solutions such as the travelingwave and kink-antikink solutions are presented probably for the first time. In Section 2.2, an ultradiscrete analogue of the sine-Gordon equation is proposed and the solutions are obtained. Because of ultradiscretization with $u \mathbb{R}$, correspondence between the discrete and ultradiscrete systems are direct, which is also supported by figures.

In Section 3.1, a noncommutative discrete analogue of the sine-Gordon equation is proposed. A relation to other integrable systems including the noncommutative discrete KP equation is 
explained, and multisoliton solutions are constructed by a repeated application of Darboux transformations. In Section 3.2, a noncommutative ultradiscrete analogue of the sine-Gordon equation is proposed and 1-soliton and 2-soliton solutions are derived. Also figures of solutions for both equations are displayed.

In Section 4, concluding remarks and discussions are presented.

\section{Discrete and ultradiscrete sine-Gordon equations}

In this section, we first review the discrete sine-Gordon equation $[3,7]$ and several results around it. Explicit calculation of the traveling-wave, kink-antikink, kink-kink, and breather solutions are probably presented for the first time.

Then, we propose an ultradiscrete analogue of the sine-Gordon equation. The solutions are obtained in two ways: by calculations completely inside $u \mathbb{R}$, and by ultradiscretization with $u \mathbb{R}$. The correspondence between the discrete and ultradiscrete systems is quite clear. Similarity of profiles of solutions is also visually confirmed by figures. Our formulation is different from Isojima et al. [9].

\subsection{Discrete sine-Gordon equation}

We review the three representations of the discrete sine-Gordon equation, their connection to the sine-Gordon equation, and some solutions for the sine-Gordon equation.

For any function $f=f(l, m)$ over $\mathbb{Z}^{2}$, define shift operations by

$$
f_{l}=f_{l}(l, m)=f(l+1, m), \quad f_{m}=f_{m}(l, m)=f(l, m+1) .
$$

Inverse operations are denoted by

$$
f_{\bar{l}}=f(l-1, m), \quad f_{\bar{m}}=f(l, m-1) .
$$

Let $\tau=\tau(l, m), \sigma=\sigma(l, m)$ be functions $\mathbb{Z}^{2} \rightarrow \mathbb{C}$. Date et al. [3] gave the discrete sine-Gordon equation $(\mathrm{ds} G)$ in the following form

$$
\begin{aligned}
& (1-\delta) \tau \tau_{l m}=\tau_{l} \tau_{m}-\delta \sigma_{l} \sigma_{m}, \\
& (1-\delta) \sigma \sigma_{l m}=\sigma_{l} \sigma_{m}-\delta \tau_{l} \tau_{m},
\end{aligned}
$$

where $\delta \in \mathbb{C}^{\times}$is a parameter with a small absolute value. The vacuum solution

$$
\tau=\sigma=1
$$

is the simplest solution, other than the null solution $\tau=\sigma=0$. Calculating the cross product of the both sides of $(2.1 \mathrm{a}),(2.1 \mathrm{~b})$, we have

$$
\tau \tau_{l m}\left(\sigma_{l} \sigma_{m}-\delta \tau_{l} \tau_{m}\right)=\sigma \sigma_{l m}\left(\tau_{l} \tau_{m}-\delta \sigma_{l} \sigma_{m}\right) \Longleftrightarrow \frac{\tau_{l m} \sigma_{m}}{\sigma_{l m} \tau_{m}}-\frac{\tau_{l} \sigma}{\sigma_{l} \tau}+\delta\left(\frac{\sigma_{m} \sigma}{\tau_{m} \tau}-\frac{\tau_{l m} \tau_{l}}{\sigma_{l m} \sigma_{l}}\right)=0
$$

and thus

$$
\frac{w_{l m}}{w_{m}}-\frac{w_{l}}{w}+\delta\left(\frac{1}{w_{m} w}-w_{l m} w_{l}\right)=0,
$$

where $w$ is defined by

$$
w=\frac{\tau}{\sigma} .
$$


If we introduce $u$ defined by

$$
u=\frac{2}{i} \log w
$$

we have

$$
\begin{aligned}
& e^{i\left(u_{l m}-u_{m}\right) / 2}-e^{i\left(u_{l}-u\right) / 2}+\delta\left(e^{i\left(-u_{m}-u\right) / 2}-e^{i\left(u_{l m}+u_{l}\right) / 2}\right)=0 \\
& \Longleftrightarrow \sin \left(\frac{u_{l m}-u_{l}-u_{m}+u}{4}\right)=\delta \sin \left(\frac{u_{l m}+u_{l}+u_{m}+u}{4}\right) .
\end{aligned}
$$

Each of (2.2) and (2.3) is also called the discrete sine-Gordon equation, where (2.3) is the original form discovered by Hirota [7].

Remark 2.1. By the non-autonomous transformation $w^{\prime}=w^{(-1)^{m}},(2.2)$ becomes

$$
\frac{w_{m}^{\prime}}{w_{l m}^{\prime}}-\frac{w_{l}^{\prime}}{w^{\prime}}+\delta\left(\frac{w_{m}^{\prime}}{w^{\prime}}-\frac{w_{l}^{\prime}}{w_{l m}^{\prime}}\right)=0 \Longleftrightarrow \frac{w_{m}^{\prime}-\delta w_{l}^{\prime}}{w_{l m}^{\prime}}=\frac{w_{l}^{\prime}-\delta w_{m}^{\prime}}{w^{\prime}} .
$$

This is known as a discrete analogue of the modified KdV equation [16], and its ultradiscretization is also known [20].

Assume $u$ is also a function $u(x, y)$ of continuum variables $x, y \in \mathbb{R}$ and has an expansion

$$
u(x+r, y+s)=u+\left(r u_{x}+s u_{y}\right)+\frac{1}{2}\left(r^{2} u_{x x}+2 r s u_{x y}+s^{2} u_{y y}\right)+\cdots,
$$

where $u_{x}=\partial u / \partial x$, etc. Connect $l, m$ to $x, y$ via the Miwa transformation

$$
u(x, y ; l, m)=u(x+l a, y+m b)
$$

where $a, b \in \mathbb{R}^{\times}$are parameters. Then we have

$$
u_{l m}-u_{l}-u_{m}+u=a b u_{x y}+(\text { higher-order terms of } a, b) .
$$

Setting $\delta=a b$ and taking the limit $a, b \rightarrow 0$ of (2.3) successively, we obtain

$$
\begin{aligned}
& \lim _{a, b \rightarrow 0} \frac{1}{a b} \sin \left(\frac{u_{l m}-u_{l}-u_{m}+u}{4}\right)=\lim _{a, b \rightarrow 0} \frac{1}{a b} \sin \left(\frac{a b u_{x y}+\cdots}{4}\right)=\frac{1}{4} u_{x y}, \\
& \lim _{a, b \rightarrow 0} \sin \left(\frac{u_{l m}+u_{l}+u_{m}+u}{4}\right)=\sin u
\end{aligned}
$$

and thus the (continuous) sine-Gordon equation

$$
u_{x y}=4 \sin u .
$$

This is known to have following special types of solutions (see, for example, [6]): the travelingwave solution

$$
u=4 \arctan \exp \left( \pm \frac{x-v y}{\sqrt{1-v^{2}}}\right)
$$

the kink-antikink solution

$$
u=4 \arctan \left(\frac{\sinh \frac{v y}{\sqrt{1-v^{2}}}}{v \cosh \frac{x}{\sqrt{1-v^{2}}}}\right),
$$


the kink-kink solution

$$
u=4 \arctan \left(\frac{v \sinh \frac{x}{\sqrt{1-v^{2}}}}{\cosh \frac{v y}{\sqrt{1-v^{2}}}}\right),
$$

and the breather solution

$$
u=4 \arctan \left(\frac{\sqrt{1-\omega^{2}}}{\omega} \frac{\sin \omega y}{\cosh \sqrt{1-\omega^{2}} x}\right),
$$

where $v, \omega$ are constants.

\subsubsection{1-soliton and 2-soliton solutions}

Isojima et al. [9] have given the following conditions for $\tau$ and $\sigma$ to be a 1-soliton or 2-soliton solution. As a 1-soliton solution, assume

$$
\tau=1+f, \quad \sigma=1-f, \quad f=c p^{l} q^{m}
$$

where $c, p, q \in \mathbb{C}^{\times}$are constants. By substitution, the dispersion relation

$$
(1-\delta)(1+p q)=(1+\delta)(p+q) \Longleftrightarrow q=\frac{(1+\delta) p-(1-\delta)}{(1-\delta) p-(1+\delta)}
$$

is found to be a necessary and sufficient condition. As a 2-soliton solution, assume

$$
\tau=1+f_{1}+f_{2}+\alpha f_{1} f_{2}, \quad \sigma=1-f_{1}-f_{2}+\alpha f_{1} f_{2}, \quad f_{j}=c_{j} p_{j}^{l} q_{j}^{m},
$$

where $\alpha \in \mathbb{C}^{\times}$is a constant. This time, the pair of the dispersion relation

$$
(1-\delta)\left(1+p_{j} q_{j}\right)=(1+\delta)\left(p_{j}+q_{j}\right) \Longleftrightarrow q_{j}=\frac{(1+\delta) p_{j}-(1-\delta)}{(1-\delta) p_{j}-(1+\delta)}
$$

and the relation

$$
\alpha=-\frac{p_{1}-p_{2}}{1-p_{1} p_{2}} \frac{q_{1}-q_{2}}{1-q_{1} q_{2}}=\left(\frac{p_{1}-p_{2}}{1-p_{1} p_{2}}\right)^{2}
$$

is a necessary and sufficient condition.

Fig. 2.1 shows the 1 -soliton solution with

$$
\delta=0.04, \quad c=-1, \quad p=2,
$$

and the 2-soliton solution with

$$
\delta=0.04, \quad c_{1}=c_{2}=-2.125, \quad p_{1}=q_{2}=2,
$$

in the light-cone coordinates

$$
(n, t)=\left(\frac{l+m}{2}, \frac{l-m}{2}\right) \Longleftrightarrow(l, m)=(n+t, n-t) .
$$



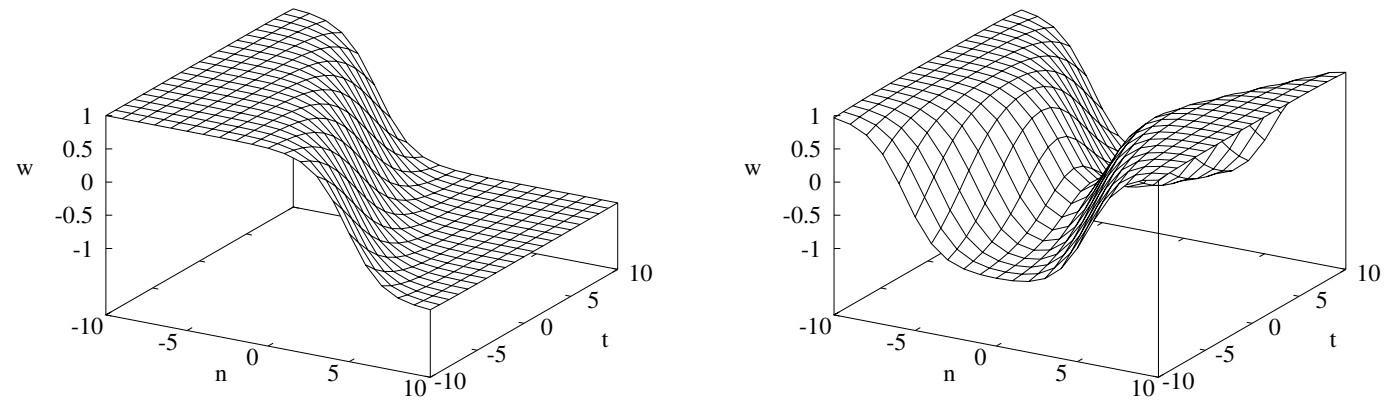

Figure 2.1. 1-soliton solution (left) and 2-soliton solution (right) for dsG.
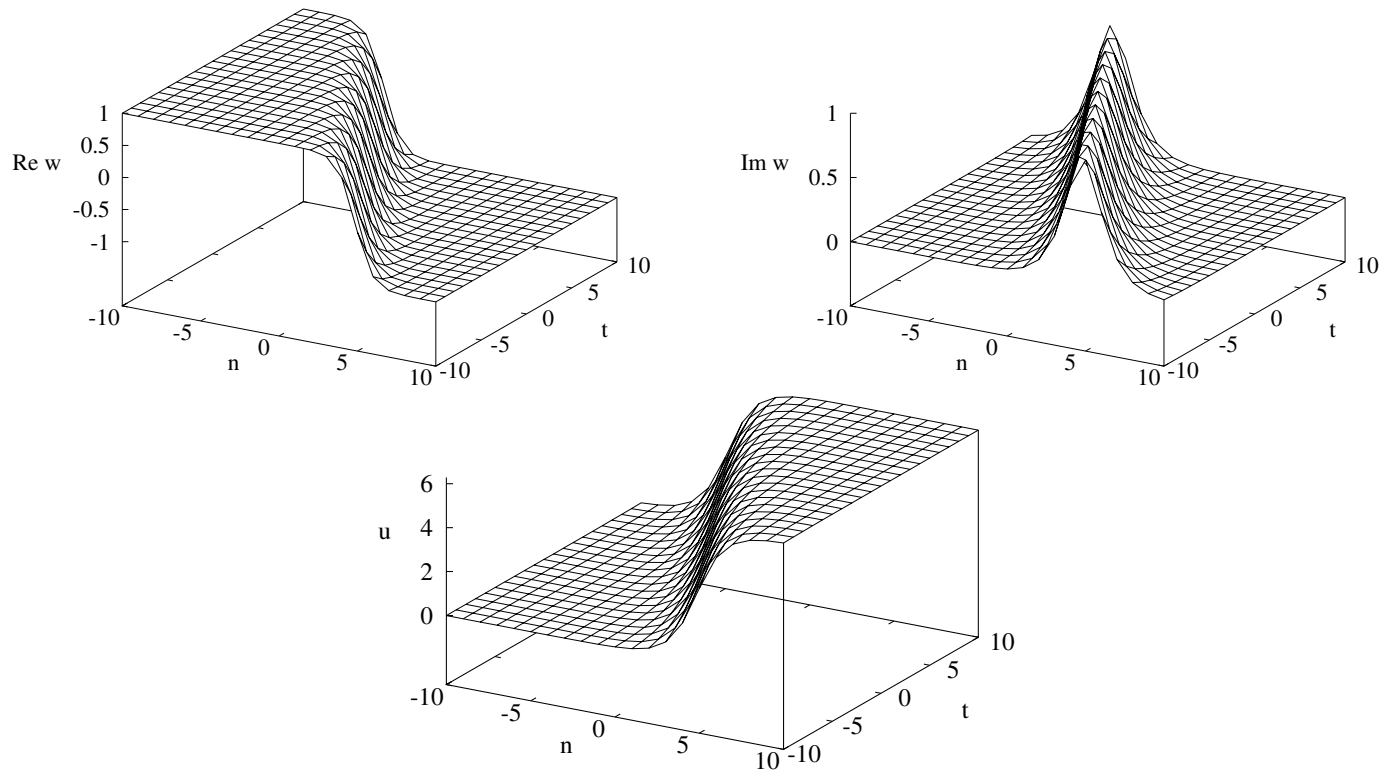

Figure 2.2. Traveling-wave solution for dsG.

\subsubsection{Traveling-wave solution}

In Sections 2.1.2-2.1.4, we give solutions for (2.1a) and (2.1b) which correspond to the continuous counterparts $(2.4)-(2.7)$. These do not seem to be previously presented in the literature, including Hirota [7] and Isojima et al. [9].

Replacing $c$ by $i c$ in the 1-soliton solution, we obtain

$$
w=\frac{1+i c(p q)^{n}\left(p q^{-1}\right)^{t}}{1-i c(p q)^{n}\left(p q^{-1}\right)^{t}}, \quad u=4 \arctan \left(c(p q)^{n}\left(p q^{-1}\right)^{t}\right)
$$

in the light-cone coordinates. This corresponds to the traveling-wave solution (2.4) for the sine-Gordon equation (if we restrict $\delta, c, p \in \mathbb{R}^{\times}$). Fig. 2.2 shows the solution with

$$
\delta=0.04, \quad c=1, \quad p=2 .
$$

\subsubsection{Kink-antikink and kink-kink solutions}

Set $p_{1}=q_{2}$ in the 2-soliton solution. Then

$$
p_{1}=\frac{(1+\delta) p_{2}-(1-\delta)}{(1-\delta) p_{2}-(1+\delta)} \Longleftrightarrow p_{2}=\frac{(1+\delta) p_{1}-(1-\delta)}{(1-\delta) p_{1}-(1+\delta)}
$$



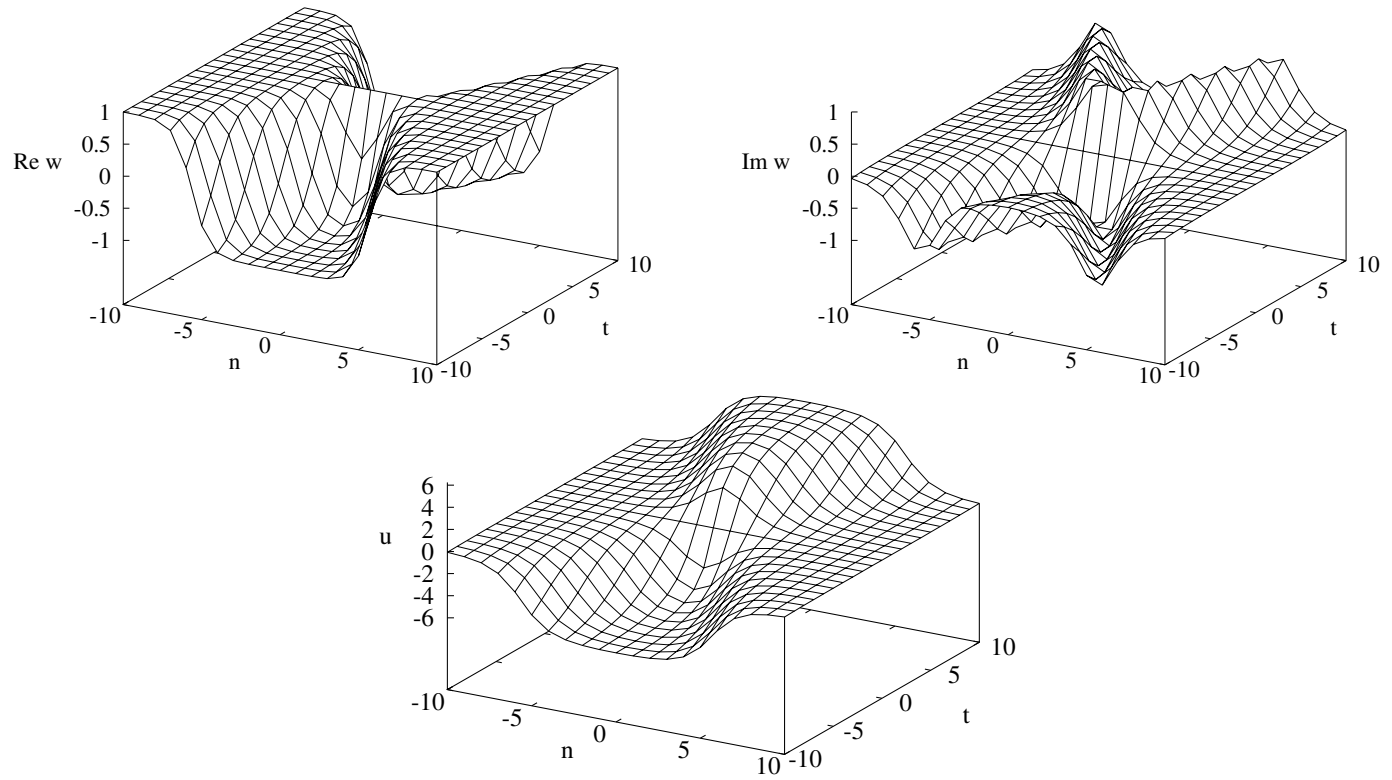

Figure 2.3. Kink-antikink solution for dsG.

and thus $p_{2}=q_{1}$. Rewriting in the light-cone coordinates, we have

$$
\begin{aligned}
\tau & =1+c_{1} p_{1}^{n+t} p_{2}^{n-t}+c_{2} p_{2}^{n+t} p_{1}^{n-t}+\alpha c_{1} c_{2}\left(p_{1} p_{2}\right)^{2 n} \\
& =\left(p_{1} p_{2}\right)^{n}\left(\alpha c_{1} c_{2}\left(p_{1} p_{2}\right)^{n}+\left(p_{1} p_{2}\right)^{-n}+c_{1}\left(p_{1} p_{2}^{-1}\right)^{t}+c_{2}\left(p_{1} p_{2}^{-1}\right)^{-t}\right), \\
\sigma & =\left(p_{1} p_{2}\right)^{n}\left(\alpha c_{1} c_{2}\left(p_{1} p_{2}\right)^{n}+\left(p_{1} p_{2}\right)^{-n}-c_{1}\left(p_{1} p_{2}^{-1}\right)^{t}-c_{2}\left(p_{1} p_{2}^{-1}\right)^{-t}\right) .
\end{aligned}
$$

We set

$$
\beta= \pm \frac{p_{1}-p_{2}}{1-p_{1} p_{2}}, \quad c_{1}=-c_{2}=i \beta^{-1}
$$

and define

$$
\operatorname{ch}(p, l)=\frac{p^{l}+p^{-l}}{2}, \quad \operatorname{sh}(p, l)=\frac{p^{l}-p^{-l}}{2} .
$$

Then

$$
w=\frac{\beta \operatorname{ch}\left(p_{1} p_{2}, n\right)+i \operatorname{sh}\left(p_{1} p_{2}^{-1}, t\right)}{\beta \operatorname{ch}\left(p_{1} p_{2}, n\right)-i \operatorname{sh}\left(p_{1} p_{2}^{-1}, t\right)}, \quad u=4 \arctan \left(\frac{\operatorname{sh}\left(p_{1} p_{2}^{-1}, t\right)}{\beta \operatorname{ch}\left(p_{1} p_{2}, n\right)}\right) .
$$

This corresponds to the kink-antikink solution (2.5). Fig. 2.3 shows the solution with

$$
\delta=0.04, \quad c_{1}=-c_{2}=2.125 i, \quad p_{1}=2 .
$$

Similarly, setting $p_{1} q_{2}=1$ gives

$$
p_{1}^{-1}=\frac{(1+\delta) p_{2}-(1-\delta)}{(1-\delta) p_{2}-(1+\delta)} \Longleftrightarrow p_{2}^{-1}=\frac{(1+\delta) p_{1}-(1-\delta)}{(1-\delta) p_{1}-(1+\delta)}
$$

and thus $p_{2} q_{1}=1$. We have

$$
\begin{aligned}
& \tau=\beta^{-1}\left(p_{1} p_{2}\right)^{t}\left(\beta\left(p_{1} p_{2}\right)^{t}+\beta\left(p_{1} p_{2}\right)^{-t}+i\left(p_{1} p_{2}^{-1}\right)^{n}-i\left(p_{1} p_{2}^{-1}\right)^{-n}\right), \\
& \sigma=\beta^{-1}\left(p_{1} p_{2}\right)^{t}\left(\beta\left(p_{1} p_{2}\right)^{t}+\beta\left(p_{1} p_{2}\right)^{-t}-i^{-1}\left(p_{1} p_{2}^{-1}\right)^{n}+i\left(p_{1} p_{2}^{-1}\right)^{-n}\right)
\end{aligned}
$$



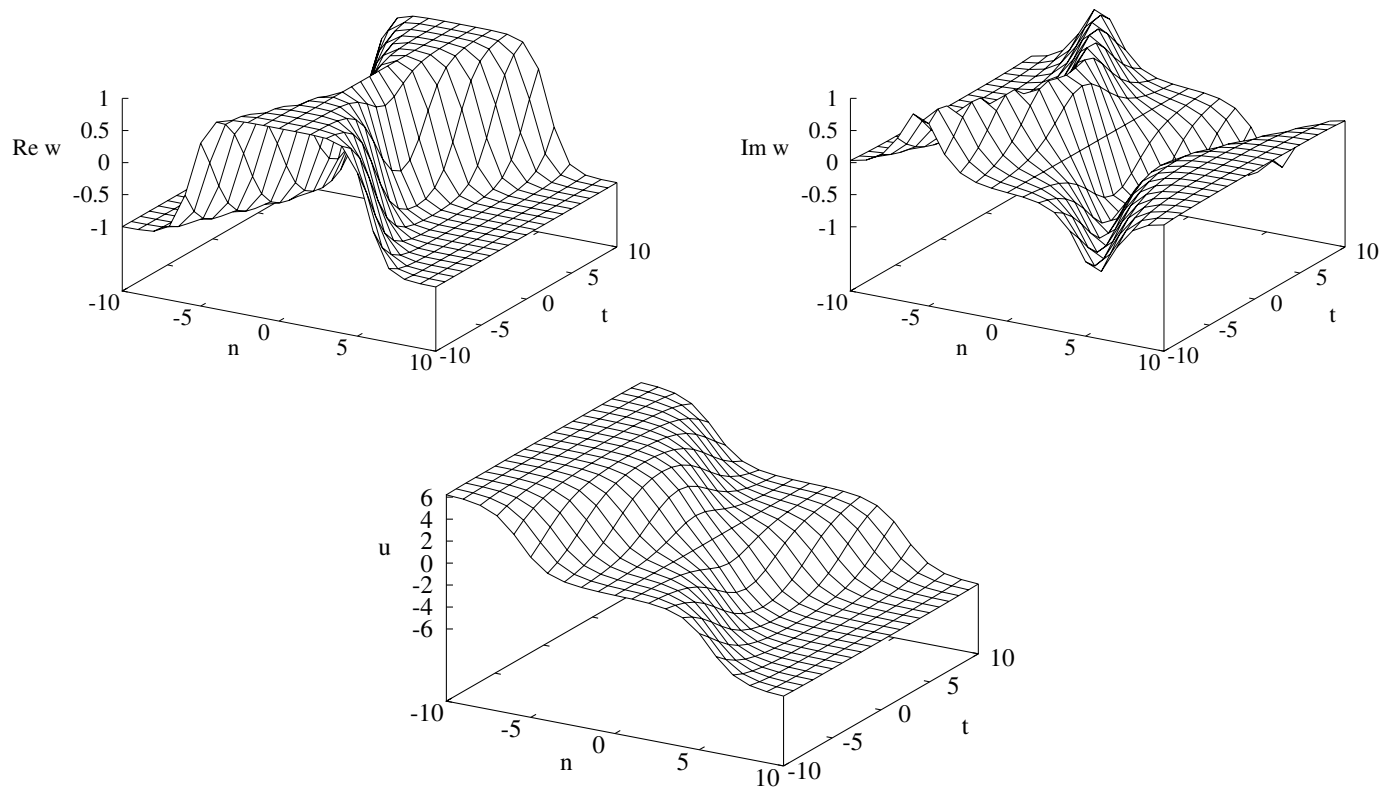

Figure 2.4. Kink-kink solution for dsG.

for the same $\beta, c_{1}, c_{2}$ defined above and

$$
w=\frac{\beta \operatorname{ch}\left(p_{1} p_{2}, t\right)+i \operatorname{sh}\left(p_{1} p_{2}^{-1}, n\right)}{\beta \operatorname{ch}\left(p_{1} p_{2}, t\right)-i \operatorname{sh}\left(p_{1} p_{2}^{-1}, n\right)}, \quad u=4 \arctan \left(\frac{\operatorname{sh}\left(p_{1} p_{2}^{-1}, n\right)}{\beta \operatorname{ch}\left(p_{1} p_{2}, t\right)}\right) .
$$

This corresponds to the kink-kink solution (2.6). Fig. 2.4 shows the solution with

$$
\delta=0.04, \quad c_{1}=-c_{2}=-0.470588 i, \quad p_{1}=2 .
$$

\subsubsection{Breather solution}

Consider the kink-antikink solution where $p_{1}$ and $p_{2}$ are complex numbers satisfying

$$
p_{1} p_{2} \in \mathbb{R}_{>0}, \quad\left|p_{1} p_{2}^{-1}\right|=1 .
$$

Such $p_{1}, p_{2}$ are complex conjugates of each other. If we write $p_{1}=g+h i, p_{2}=g-h i$ and substitute these into (2.13), we find $g, h$ must satisfy

$$
(1-\delta)\left(1+g^{2}+h^{2}\right)=2(1+\delta) g \Longleftrightarrow(1-\delta) g^{2}-2(1+\delta) g+(1-\delta)\left(1+h^{2}\right)=0 .
$$

As a quadratic equation of $g$, the condition for the existence of real roots is given by

$$
(1+\delta)^{2}-(1-\delta)^{2}\left(1+h^{2}\right) \geq 0 \Longleftrightarrow h^{2} \leq\left(\frac{1+\delta}{1-\delta}\right)^{2}-1
$$

Such a real number $h$ does exist if $\delta \geq 0$, and $g$ is given by

$$
g=\frac{1+\delta}{1-\delta} \pm \sqrt{\left(\frac{1+\delta}{1-\delta}\right)^{2}-\left(1+h^{2}\right)} .
$$

Rewriting $p_{1}=r e^{i \theta}, p_{2}=r e^{-i \theta}$, we obtain $\beta=i \gamma$ where $\gamma$ is defined by

$$
\gamma= \pm \frac{2 r \sin \theta}{1-r^{2}}
$$



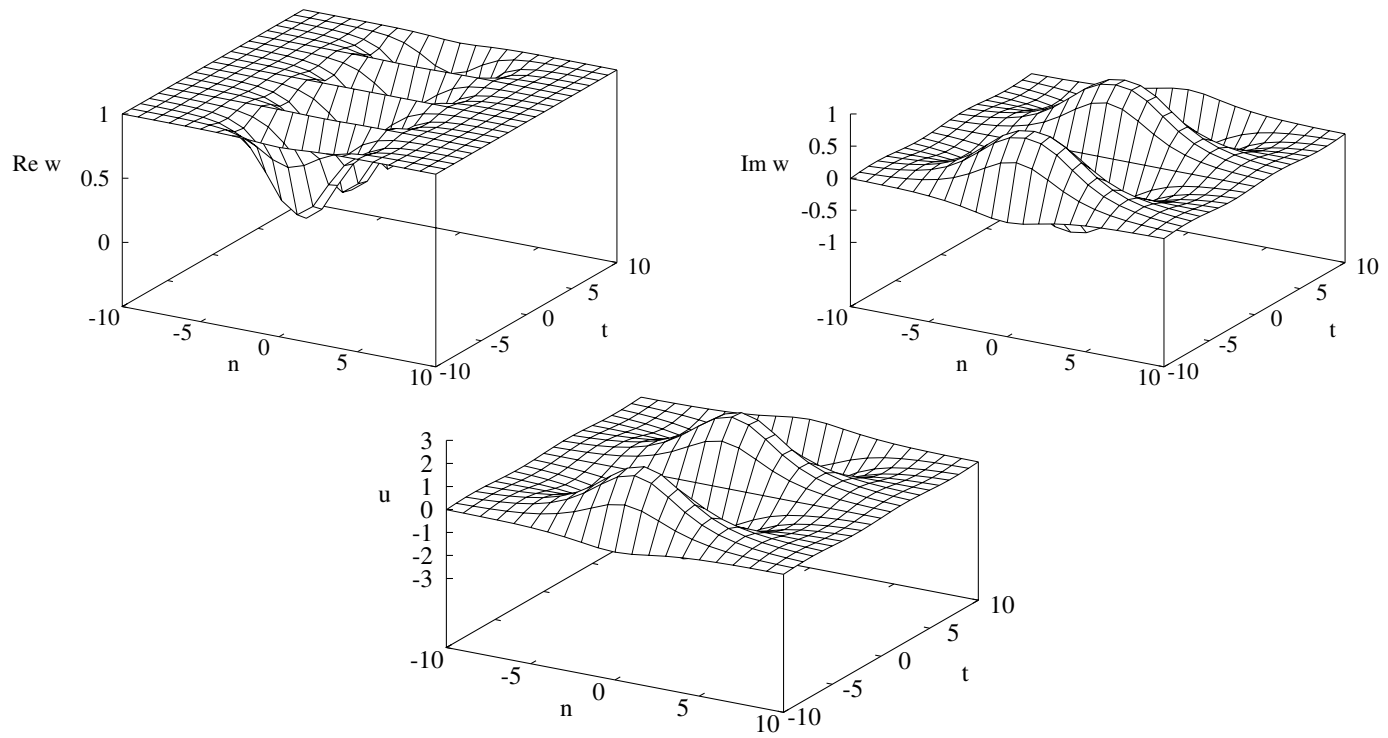

Figure 2.5. Breather solution for dsG.

and $\operatorname{sh}\left(p_{1} p_{2}^{-1}, t\right)=i \sin 2 t \theta$. Thus,

$$
w=\frac{\gamma \operatorname{ch}\left(r^{2}, n\right)+i \sin 2 t \theta}{\gamma \operatorname{ch}\left(r^{2}, n\right)-i \sin 2 t \theta}, \quad u=4 \arctan \left(\frac{\sin 2 t \theta}{\gamma \operatorname{ch}\left(r^{2}, n\right)}\right) .
$$

This corresponds to the breather solution (2.7). Fig. 2.5 shows the solution with

$$
\delta=0.04, \quad c_{1}=-c_{2}=0.75, \quad p_{1}=0.75+0.25 i .
$$

\subsection{Ultradiscrete sine-Gordon equation}

\subsubsection{Ultradiscrete sine-Gordon equation}

In order to ultradiscretize the discrete sine-Gordon equation (2.1a), (2.1b), we must deal with negative numbers since either or both of $\tau$ and $\sigma$ include subtractions. We adopt ultradiscretization with the symmetrized max-plus algebra $u \mathbb{R}$. For details, see Appendix A and references cited there.

We perform ultradiscretization of dsG through the parametrization

$$
\delta=\mu_{D} e^{\widetilde{D} s}, \quad \widetilde{D}<0 .
$$

This can be regarded as an other aspect of continuum limit since $\delta \rightarrow 0$ as $s \rightarrow \infty$. Assuming $\delta \stackrel{\mathrm{ud}}{\longrightarrow} D, \tau \stackrel{\mathrm{ud}}{\longrightarrow} T, \sigma \stackrel{\mathrm{ud}}{\longrightarrow} S$, we obtain

$$
\begin{aligned}
& T T_{l m} \nabla T_{l} T_{m} \ominus D S_{l} S_{m}, \\
& S S_{l m} \nabla S_{l} S_{m} \ominus D T_{l} T_{m} .
\end{aligned}
$$

We call the pair (2.17a), (2.17b) the ultradiscrete sine-Gordon equation (udsG). The vacuum solution $T=S=0$ is the simplest solution, other than the null solution $T=S=-\infty$. We can also ultradiscretize $(2.2)$ to obtain

$$
W_{l m} W_{m}^{-1} \ominus W_{l} W^{-1} \oplus D\left(W_{m}^{-1} W^{-1} \ominus W_{l m} W_{l}\right) \nabla-\infty
$$

where $w \stackrel{\text { ud }}{\longrightarrow} W \nabla T S^{-1}$. We also call (2.18) the ultradiscrete sine-Gordon equation. The ultradiscretization of (2.3) is unclear. 


\subsubsection{Deterministic time evolution and class of solutions}

It seems sensible to restrict ourselves to the class of signed solutions, that is, $T, S, W \in \mathrm{u} \mathbb{C}^{\vee}$ for any $(l, m) \in \mathbb{Z}^{2}$ since it permits basic properties like weak substitution. The null and vacuum solutions are signed solutions.

The problem is that udsG no longer admits time evolution, at least deterministic one, in general, since the balance relation is not equality. For example, if we have

$$
f(t+1) \nabla(\text { expression including } f(t))=3^{\bullet},
$$

we cannot determine $f(t+1)$ from $f(t)$, since this relation is satisfied whenever $|f(t+1)|_{\oplus} \leq 3$. Strictly speaking, udsG is not an equation.

But in some cases, it actually becomes an equation, or furthermore, a deterministically evolutionary form. Multiplying $T^{-1}$ to $(2.17 \mathrm{a})$ and $S^{-1}$ to $(2.17 \mathrm{~b})$, we have

$$
T^{-1} T T_{l m} \nabla T^{-1}\left(T_{l} T_{m} \ominus D S_{l} S_{m}\right), \quad S^{-1} S S_{l m} \nabla S^{-1}\left(S_{l} S_{m} \ominus D T_{l} T_{m}\right) .
$$

If $T^{-1} T=S^{-1} S=0$ and the right hand sides are signed, we obtain

$$
\begin{aligned}
& T_{l m}=T^{-1}\left(T_{l} T_{m} \ominus D S_{l} S_{m}\right), \\
& S_{l m}=S^{-1}\left(S_{l} S_{m} \ominus D T_{l} T_{m}\right)
\end{aligned}
$$

by reduction of balances (see Appendix A). We call (2.19a), (2.19b) the deterministically evolutionary form of udsG. If we replace $D$ by $\ominus D$ and restrict ranges of $D, T, S$ to $\mathbb{R}$ for example, the assumptions are satisfied, and we obtain the completely ordinary-looking ultradiscrete equation:

$$
\begin{aligned}
& T_{l m}=\max \left(T_{l}+T_{m}, D+S_{l}+S_{m}\right)-T, \\
& S_{l m}=\max \left(S_{l}+S_{m}, D+T_{l}+T_{m}\right)-S .
\end{aligned}
$$

Deterministic time evolution is also possible in other settings, which are presented in the following sections.

It might be natural to think we should consider (2.19a), (2.19b), or even (2.20a), (2.20b) only. However, it seems that the former cannot capture the traveling-wave, kink-antikink, and kink-kink solutions. And the latter does not even seem to contain soliton solutions. Therefore, we consider $(2.17 \mathrm{a}),(2.17 \mathrm{~b})$ primarily.

We show one example of positive $(\mathbb{R})$ time evolution by (2.20a), (2.20b) in Fig. 2.6. Initial values are set as

$$
T(l,-10)=l, \quad S(-10, m)=m, \quad-10 \leq l, m \leq 10
$$

with $D=-1$. The sign (in the sense of \pm ) of the solution alternates with time.

\subsubsection{1-soliton solution}

Consider a signed solution $T, S$ satisfying

$$
T \nabla 0 \oplus F, \quad S \nabla 0 \ominus F, \quad F=C P^{l} Q^{m},
$$

where $C \in \mathrm{u} \mathbb{C}^{\vee}$ and $P, Q \in u \mathbb{R}^{\otimes}$. Weakly substituting these into (2.17a), (2.17b), we have

$$
\begin{aligned}
& 0 \oplus P Q F^{2} \oplus(0 \oplus P Q) F \nabla 0 \oplus P Q F^{2} \oplus(P \oplus Q) F, \\
& 0 \oplus P Q F^{2} \ominus(0 \oplus P Q) F \nabla 0 \oplus P Q F^{2} \ominus(P \oplus Q) F,
\end{aligned}
$$




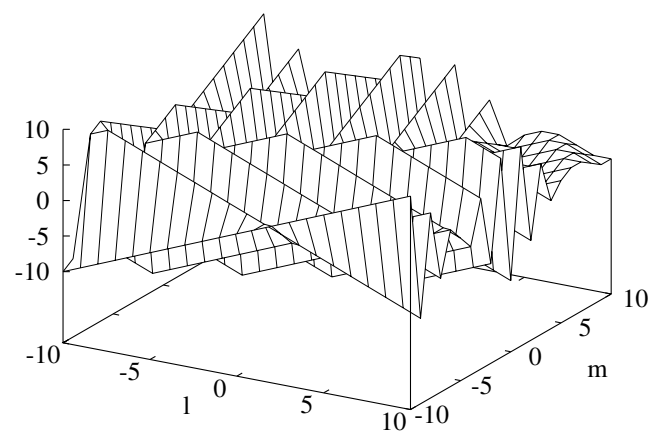

Figure 2.6. Positive $(\mathbb{R})$ evolution of udsG.

where $0 \oplus D=0 \ominus D=0$ is used. The dispersion relation

$$
0 \oplus P Q \nabla P \oplus Q
$$

is a sufficient condition for $(2.22 \mathrm{a}),(2.22 \mathrm{~b})$ to hold, since we can construct them by adding and multiplying same numbers to the both sides of (2.23). Rewriting (2.23), we have

$$
(P \ominus 0) Q \nabla(P \ominus 0)
$$

and thus $P=0$ or $Q=0$. Obviously, (2.21) and (2.23) can be obtained by ultradiscretizing (2.8) and (2.9), respectively, through

$$
c=\mu_{C} e^{\widetilde{C} s} \stackrel{\mathrm{ud}}{\longrightarrow} C, \quad p=\mu_{P} e^{\widetilde{P} s} \stackrel{\mathrm{ud}}{\longrightarrow} P, \quad q \stackrel{\mathrm{ud}}{\longrightarrow} Q
$$

or

$$
c=\mu_{C} e^{\widetilde{C} s} \stackrel{\mathrm{ud}}{\longrightarrow} C, \quad p \stackrel{\mathrm{ud}}{\longrightarrow} P, \quad q=\mu_{Q} e^{\widetilde{Q} s} \stackrel{\mathrm{ud}}{\longrightarrow} Q .
$$

The solution is, however, not completely determined yet, because the balance relation is not equality as stated before. So we try to utilize reduction of balances. If $C \in \mathrm{u} \mathbb{Z}$ is an odd number and $P, Q \in \mathrm{u} \mathbb{Z}$ are even numbers, then $F$ is always odd and $0 \oplus F, 0 \ominus F$ can never be balanced since 0 is even. By reduction of balances, we obtain

$$
T=0 \oplus F, \quad S=0 \ominus F,
$$

and $W$ is also immediately determined since $S^{-1}$ is signed. This solution admits deterministic time evolution since

$$
\left|T_{l} T_{m}\right|_{\oplus}>\left|D S_{l} S_{m}\right|_{\oplus}, \quad\left|S_{l} S_{m}\right|_{\oplus}>\left|D T_{l} T_{m}\right|_{\oplus}
$$

and thus

$$
\begin{aligned}
& T^{-1}\left(T_{l} T_{m} \ominus D S_{l} S_{m}\right)=T^{-1} T_{l} T_{m} \in u \mathbb{R}^{\otimes}, \\
& S^{-1}\left(S_{l} S_{m} \ominus D T_{l} T_{m}\right)=S^{-1} S_{l} S_{m} \in u \mathbb{R}^{\otimes} .
\end{aligned}
$$

Fig. 2.7 shows the solution with

$$
D=-1, \quad C=\ominus 1, \quad P=2
$$

in the light-cone coordinates (2.11). It is somehow difficult to depict ultradiscrete numbers in figures; here signs and absolute values are displayed separately, and signs are mapped from $\ominus 0,0^{\bullet}, \oplus 0$ to $-1,0,1$, respectively (balanced elements do not appear in the figure, though). Observe that the form of the 1-soliton solution $w$ for dsG is preserved in the signs. Absolute values are always 0 , corresponding to the fact that $w$ asymptotically behaves as \pm 1 . 

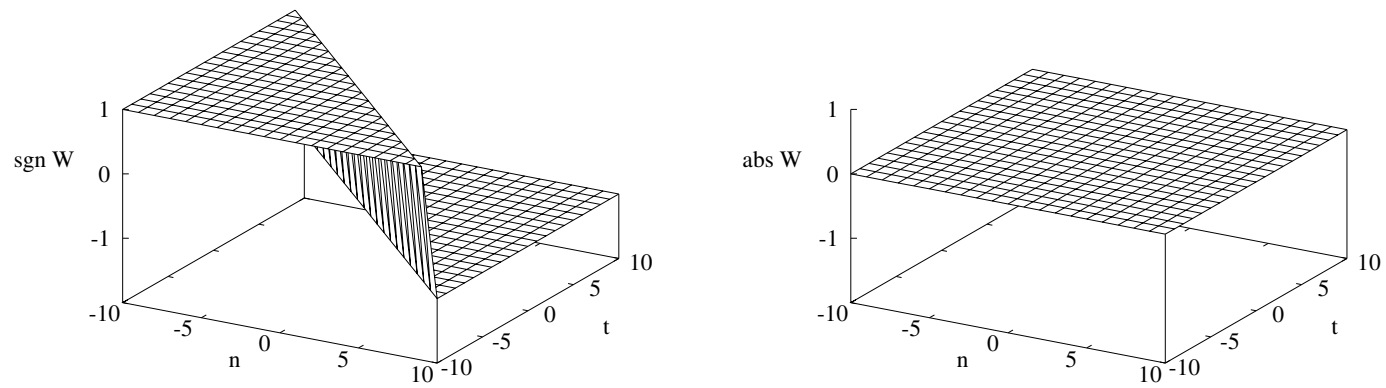

Figure 2.7. Signs (left) and absolute values (right) of 1-soliton solution for udsG.
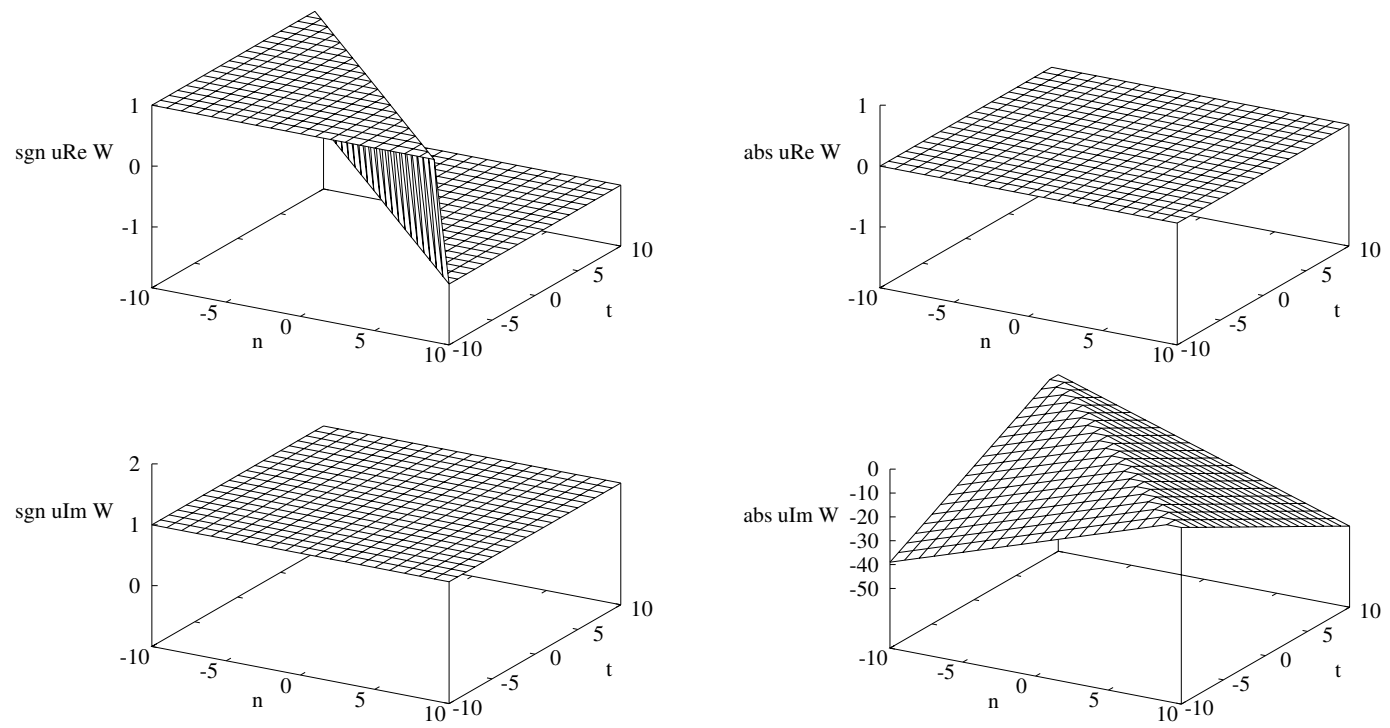

Figure 2.8. Traveling-wave solution for udsG.

\subsubsection{Traveling-wave solution}

If we replace $C$ by $C I$ and redefine $F=C P^{l} Q^{m}\left(C \in u \mathbb{R}^{\otimes}\right)$, we obtain

$$
T=0 \oplus F I, \quad S=0 \ominus F I
$$

and

$$
W \nabla \frac{\left(0 \ominus F^{2}\right) \oplus F I}{0 \oplus F^{2}} .
$$

We choose odd $C$ and even $P, Q$ so that $0 \ominus F^{2}$ is always signed and reduction of balances can be applied. This solution no longer admits deterministic time evolution, but is apparently ultradiscretization of the traveling-wave solution (2.12) for dsG. Fig. 2.8 shows the solution with

$$
D=-1, \quad C=1, \quad P=2 .
$$

The uRe and $\mathrm{uIm}$ parts are displayed separately. The profile of the traveling-wave solution for $\mathrm{dsG}$ is preserved well.

\subsubsection{2-soliton solution}

Assume

$$
T \nabla 0 \oplus F_{1} \oplus F_{2} \oplus A F_{1} F_{2}, \quad S \nabla 0 \ominus F_{1} \ominus F_{2} \oplus A F_{1} F_{2},
$$



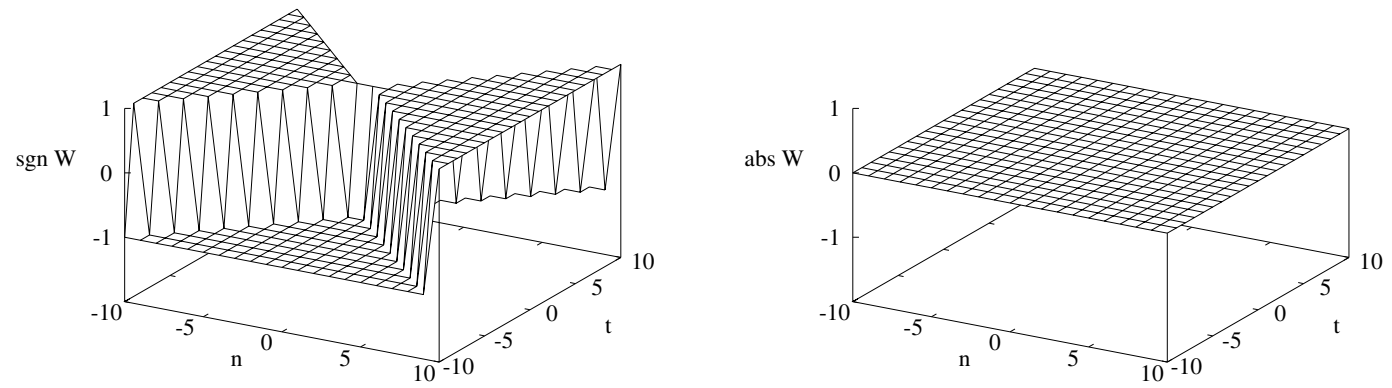

Figure 2.9. 2-soliton solution for udsG.

where $A \in \mathrm{u} \mathbb{R}^{\otimes}$ and $F_{j}=C_{j} P_{j}^{l} Q_{j}^{m}$. We also assume

$P_{1} \neq P_{2}, \quad Q_{1} \neq Q_{2}$.

By substitution, we find the pair of the dispersion relation

$$
0 \oplus P_{j} Q_{j} \nabla P_{j} \oplus Q_{j}
$$

and the relation

$$
A\left(0 \ominus P_{1} P_{2}\right)\left(0 \ominus Q_{1} Q_{2}\right) \oplus\left(P_{1} \ominus P_{2}\right)\left(Q_{1} \ominus Q_{2}\right) \nabla-\infty
$$

is a sufficient condition for (2.24) to become a solution. Obviously, (2.25) is ultradiscretization of (2.10). When $P_{1}=P_{2}=0$ or $Q_{1}=Q_{2}=0$, any $A$ satisfies (2.25). When $P_{1}=Q_{2}=0$, we have

$$
A\left(0 \ominus P_{2}\right)\left(0 \ominus Q_{1}\right) \oplus\left(0 \ominus P_{2}\right)\left(Q_{1} \ominus 0\right) \nabla-\infty \Longrightarrow A=0 .
$$

The case $P_{2}=Q_{1}=0$ is similar.

We can choose $A, C_{j}, P_{j}, Q_{j} \in \mathrm{u} \mathbb{Z}$ such that $0 \oplus A F_{1} F_{2}$ is always positive, even and $F_{1} \oplus F_{2}$ is negative, odd. Then the solution is determined as

$$
T=0 \oplus F_{1} \oplus F_{2} \oplus A F_{1} F_{2}, \quad S=0 \ominus F_{1} \ominus F_{2} \oplus A F_{1} F_{2} .
$$

This admits deterministic time evolution, of course. Fig. 2.9 shows the solution with

$$
D=-1, \quad C_{1}=C_{2}=\ominus 1, \quad P_{1}=Q_{2}=4 .
$$

\subsubsection{Kink-antikink and kink-kink solutions}

If we replace $C_{1}$ by $C_{1} I, C_{2}$ by $\ominus C_{2} I$, and redefine $F_{j}=C_{j} P_{j}^{l} Q_{j}^{m}\left(C_{j} \in \mathrm{u} \mathbb{R}^{\otimes}\right)$ in the 2-soliton solution, we obtain

$$
T \nabla 0 \oplus A F_{1} F_{2} \oplus\left(F_{1} \ominus F_{2}\right) I, \quad S \nabla 0 \oplus A F_{1} F_{2} \ominus\left(F_{1} \ominus F_{2}\right) I
$$

and

$$
W \nabla \frac{\left(\left(0 \oplus A F_{1} F_{2}\right)^{2} \ominus\left(F_{1} \ominus F_{2}\right)^{2}\right) \oplus\left(0 \oplus A F_{1} F_{2}\right)\left(F_{1} \ominus F_{2}\right) I}{\left(0 \oplus A F_{1} F_{2}\right)^{2} \oplus\left(F_{1} \ominus F_{2}\right)^{2}} .
$$

We choose $C_{j}, P_{j}, Q_{j} \in \mathrm{u} \mathbb{Z}$ such that

$$
\left|F_{1}\right|_{\oplus} \equiv 1, \quad\left|F_{2}\right|_{\oplus} \equiv 3 \quad(\bmod 4)
$$



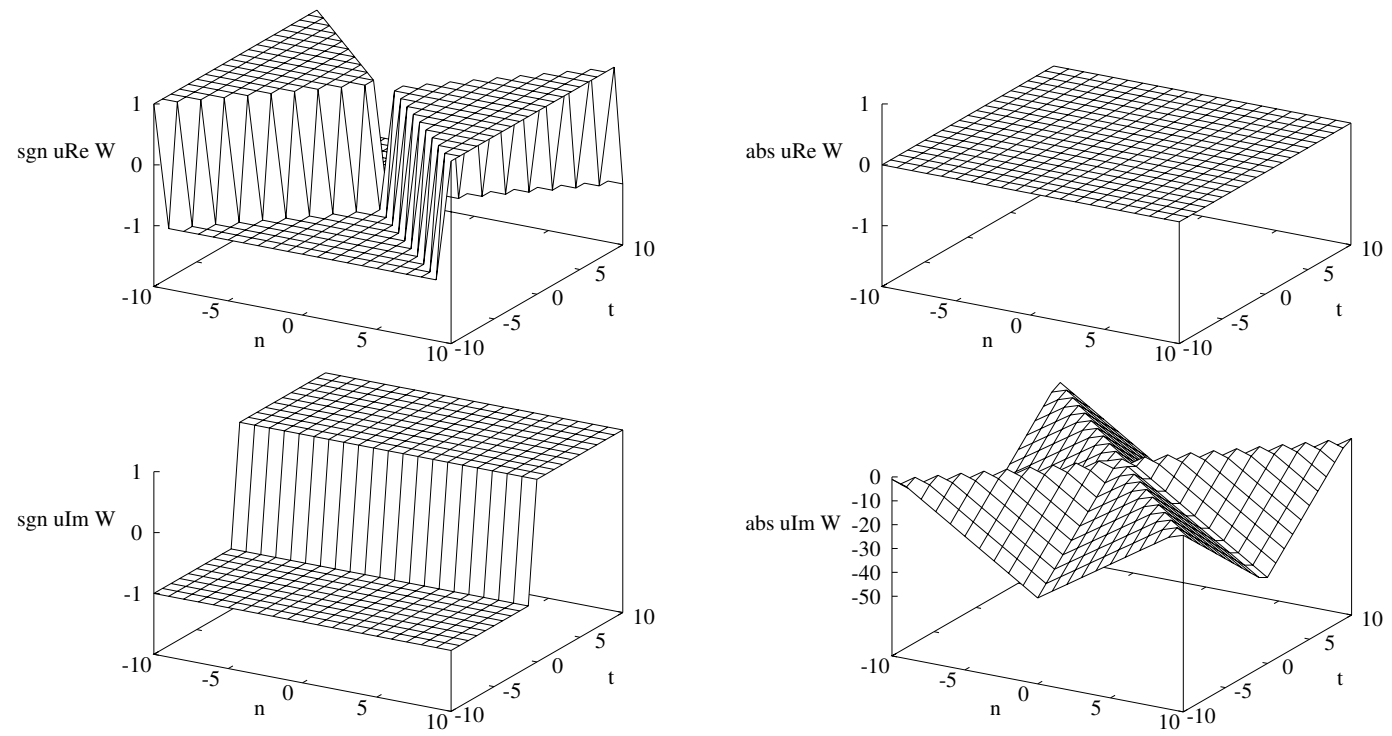

Figure 2.10. Kink-antikink solution for udsG.

or

$$
\left|F_{1}\right|_{\oplus} \equiv 3, \quad\left|F_{2}\right|_{\oplus} \equiv 1 \quad(\bmod 4) .
$$

Then $F_{1} \ominus F_{2}$ and $\left(0 \oplus A F_{1} F_{2}\right)^{2} \ominus\left(F_{1} \ominus F_{2}\right)^{2}$ are always signed and the balance relations become equalities.

If we set $P_{1}=Q_{2}, P_{2}=Q_{1}$, we have the kink-antikink solution. Similarly, setting $P_{1}=Q_{2}^{-1}$, $P_{2}=Q_{1}^{-1}$ gives the kink-kink solution. These solutions does not admit deterministic time evolution, but are ultradiscretization of (2.14), (2.15). The ultradiscretization of the breather solution is unclear.

Fig. 2.10 shows the kink-antikink solution with

$$
D=-1, \quad C_{1}=1, \quad C_{2}=-1, \quad P_{1}=Q_{2}=4,
$$

and Fig. 2.11 shows the kink-kink solution with

$$
D=-1, \quad C_{1}=\ominus 1, \quad C_{2}=\ominus(-1), \quad P_{1}=Q_{2}^{-1}=4 .
$$

Observe that in the uIm part, two waves approach to each other for $t<0$, collide at $t=0$, and move away from each other for $t>0$. In the kink-antikink solution, the two waves have the same sign and bump up by collision. In the kink-kink solution, the two have opposite signs and reflect by collision.

\section{Noncommutative discrete and ultradiscrete sine-Gordon equations}

In this section, we propose a noncommutative discrete analogue of the sine-Gordon equation as a compatibility condition of a certain linear system. This equation reduces to the commutative version once the underlying algebra turns out to be commutative and one simple reduction condition is applied. A reduction from the noncommutative discrete KP equation [12, 17] also gives the equation, and continuum limit of the equation gives the noncommutative (continuous) sineGordon equation, which is already known in a different context [14]. We define the Darboux 

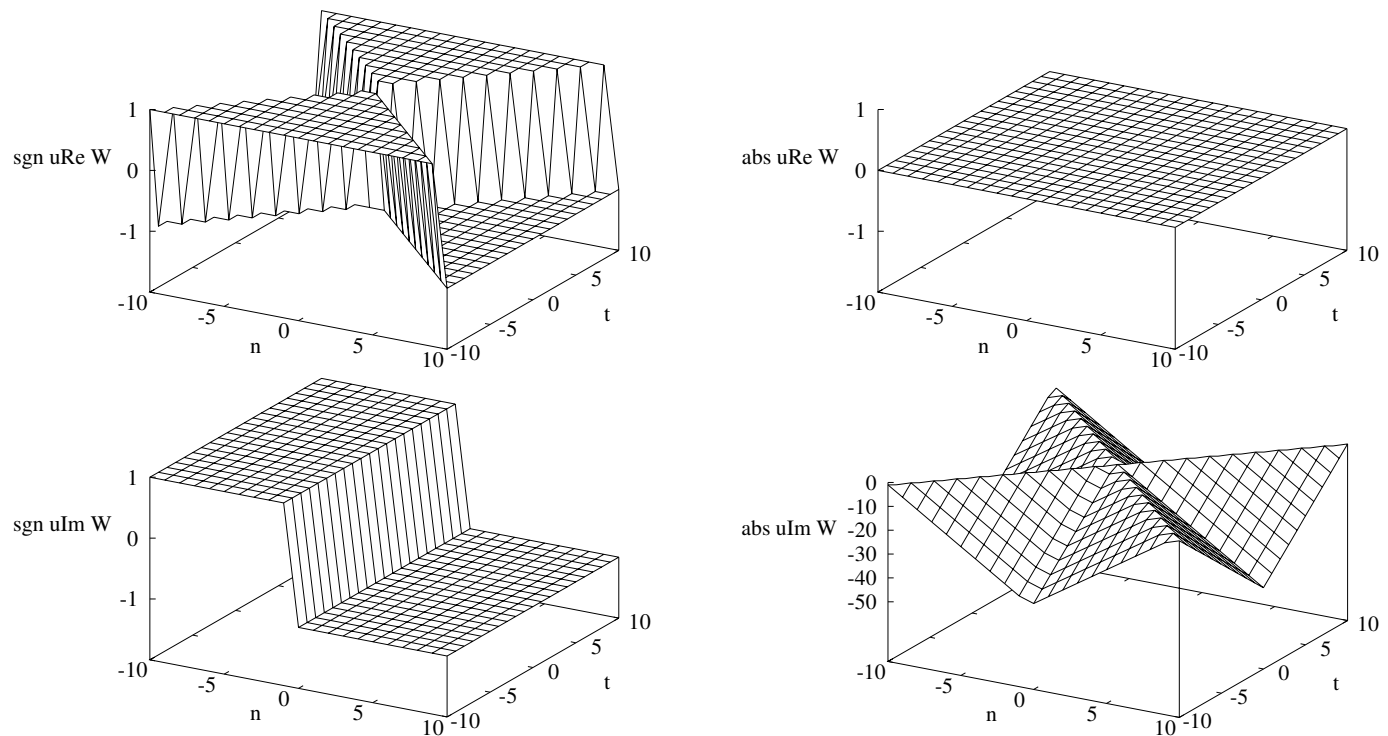

Figure 2.11. Kink-kink solution for udsG.

transformation, which constructs new solutions from old ones, and obtain Casoratian-type solutions by repeating it. Explicitly setting the starting solutions for repetition, we derive so-called multisoliton solutions.

Along the construction of Casoratian-type solutions, quasideterminants [5] are used, which is a noncommutative extension of determinants. The theory needs some space for explanation, but it is not essential to the main story. Therefore, we only briefly explain the definition and some properties of them in Appendix B. For details, see [5].

We finally propose a noncommutative ultradiscrete analogue of the sine-Gordon equation. Noncommutative ultradiscrete setting is probably one of the hardest environments for integrable systems to exist, but we manage to obtain 1-soliton and 2-soliton solutions by ultradiscretization.

Notations are slightly changed in this section because of the complexity of expressions we are going to manipulate. Shifts are always indicated after a comma like $f_{, l}$. This is to distinguish indices and shifts. In addition, shift operators $T_{l}, T_{m}$ are also used:

$$
T_{l} f=f_{, l}=f(l+1, m), \quad T_{m} f=f_{, m}=f(l, m+1) .
$$

Do not confuse these with the ultradiscretized $\tau$ function of the previous section; in the noncommutative setting, $\tau$ functions do not seem to exist. We also use superscripts for elements of matrices. For example,

$$
w=\left(w^{\iota \kappa}\right)=\left(\begin{array}{ccc}
w^{11} & \cdots & w^{1 N} \\
\vdots & \ddots & \vdots \\
w^{N 1} & \cdots & w^{N N}
\end{array}\right)
$$

\subsection{Noncommutative discrete sine-Gordon equation}

\subsubsection{Linear system}

Let $w=w(l, m), v=v(l, m)$ be functions $\mathbb{Z}^{2} \rightarrow \operatorname{Mat}(N, \mathbb{C})$ and

$$
B_{l}=\left(\begin{array}{cc}
w_{, l} w^{-1} & -a \lambda \\
-a \lambda & v_{, l} v^{-1}
\end{array}\right), \quad B_{m}=\left(\begin{array}{cc}
1 & -b \lambda^{-1} w_{, m} v^{-1} \\
-b \lambda^{-1} v_{, m} w^{-1} & 1
\end{array}\right)
$$


where $a, b, \lambda \in \mathbb{C}^{\times}$are parameters. Consider the linear system

$$
T_{l}\left(\begin{array}{l}
\phi \\
\psi
\end{array}\right)=B_{l}\left(\begin{array}{l}
\phi \\
\psi
\end{array}\right), \quad T_{m}\left(\begin{array}{c}
\phi \\
\psi
\end{array}\right)=B_{m}\left(\begin{array}{l}
\phi \\
\psi
\end{array}\right)
$$

for $\phi, \psi: \mathbb{Z}^{2} \rightarrow \operatorname{Mat}(N, \mathbb{C})$. Denoting entrywise shift operations by $T_{m} B_{l}=B_{l, m}$ etc., we have

$$
\begin{aligned}
& T_{m} T_{l}\left(\begin{array}{l}
\phi \\
\psi
\end{array}\right)=B_{l, m} T_{m}\left(\begin{array}{l}
\phi \\
\psi
\end{array}\right)=B_{l, m} B_{m}\left(\begin{array}{l}
\phi \\
\psi
\end{array}\right), \\
& T_{l} T_{m}\left(\begin{array}{c}
\phi \\
\psi
\end{array}\right)=B_{m, l} T_{l}\left(\begin{array}{c}
\phi \\
\psi
\end{array}\right)=B_{m, l} B_{l}\left(\begin{array}{l}
\phi \\
\psi
\end{array}\right) .
\end{aligned}
$$

These must coincide, so we require the compatibility condition

$$
B_{l, m} B_{m}=B_{m, l} B_{l} .
$$

This is equivalent to

$$
\begin{aligned}
& w_{, l m} w_{, m}^{-1}-w_{, l} w^{-1}+a b\left(v_{, m} w^{-1}-w_{, l m} v_{, l}^{-1}\right)=0 \\
& v_{, l m} v_{, m}^{-1}-v_{, l} v^{-1}+a b\left(w_{, m} v^{-1}-v_{, l m} w_{, l}^{-1}\right)=0 .
\end{aligned}
$$

We call the pair (3.2a) and (3.2b) the noncommutative discrete sine-Gordon equation (ncdsG).

Proposition 3.1. When $N=1$, the reduction condition

$$
w v=1
$$

gives the (commutative) discrete sine-Gordon equation $[3,7]$

$$
\frac{w_{, l m}}{w_{, m}}-\frac{w_{, l}}{w}+a b\left(\frac{1}{w_{, m} w}-w_{, l m} w_{, l}\right)=0 .
$$

Proof. Under (3.3), (3.2a) is apparently equivalent to (3.4). Since

$$
\begin{aligned}
(\text { l.h.s. of }(3.2 \mathrm{a})) \times\left(w_{, m} w-\frac{1}{w_{, l m} w_{, l}}\right) & =w_{, l m} w+\frac{1}{w_{, l m} w}-w_{, l} w_{, m}-\frac{1}{w_{, l} w_{, m}} \\
& =(\text { l.h.s. of }(3.2 \mathrm{~b})) \times\left(\frac{1}{w_{, m} w}-w_{, l m} w_{, l}\right),
\end{aligned}
$$

(3.2b) is also equivalent to (3.4).

For any $w_{0}$ satisfying

$$
a b w_{0, \bar{l} m}=\frac{w_{0, l \bar{m}}}{a b}
$$

(3.2a) and (3.2b) are solved by $(w, v)=\left(w_{0}, a b w_{0, \bar{l} m}\right)$, which is not an interesting solution. We consider other types of solutions in the rest of this section. 


\subsubsection{Reduction from the noncommutative discrete KP equation}

Let $w_{i}=w_{i}\left(n_{1}, n_{2}, n_{3}\right)(i=1,2,3)$ be functions $\mathbb{Z}^{3} \rightarrow \operatorname{Mat}(N, \mathbb{C})$. The noncommutative discrete $\mathrm{KP}$ equation $[12,17]$ is the set of equations

$$
w_{i, j}\left(c_{i}-c_{j}\right) w_{i}^{-1}+w_{j, k}\left(c_{j}-c_{k}\right) w_{j}^{-1}+w_{k, i}\left(c_{k}-c_{i}\right) w_{k}^{-1}=0
$$

for any combination of $i, j, k \in\{1,2,3\}$. Here $i, j, k$ can take same values, and shifts are denoted like

$$
w_{1,2}=w_{1}\left(n_{1}, n_{2}+1, n_{3}\right) .
$$

$c_{i} \in \mathbb{C}^{\times}$are parameters taking mutually different values.

We replace $\left(n_{1}, n_{2}, n_{3}\right)$ by new coordinates $\left(n_{1}^{\prime}, n_{2}^{\prime}, n_{3}^{\prime}\right)=\left(n_{1}-n_{3}, n_{2}, n_{3}\right)$. Shifts are also in new coordinates, and double-shifts are to be used:

$$
w_{1,2}=w_{1}\left(n_{1}^{\prime}, n_{2}^{\prime}+1, n_{3}^{\prime}\right), \quad w_{2,13}=w_{2}\left(n_{1}^{\prime}+1, n_{2}^{\prime}, n_{3}^{\prime}+1\right), \quad \text { etc. }
$$

Then, setting

$$
\delta=\frac{c_{1}-c_{3}}{c_{1}-c_{2}}
$$

we can rewrite $(3.5)$ as

$$
\begin{aligned}
& w_{1,2} w_{1}^{-1}+(\delta-1) w_{2,13} w_{2}^{-1}-\delta w_{3,1} w_{3}^{-1}=0 \\
& w_{1,2} w_{1}^{-1}=w_{2,1} w_{2}^{-1}, \quad w_{2,13} w_{2}^{-1}=w_{3,2} w_{3}^{-1}, \quad w_{3,1} w_{3}^{-1}=w_{1,13} w_{1}^{-1} .
\end{aligned}
$$

By imposing the reduction condition

$$
w_{i}\left(n_{1}^{\prime}+2, n_{2}^{\prime}, n_{3}^{\prime}\right)=w_{i}\left(n_{1}^{\prime}, n_{2}^{\prime}, n_{3}^{\prime}\right)
$$

and defining $v_{i}=w_{i, 1}$, we obtain

$$
\begin{aligned}
& w_{1,2} w_{1}^{-1}+(\delta-1) v_{2,3} w_{2}^{-1}-\delta v_{3} w_{3}^{-1}=0 \\
& v_{1,2} v_{1}^{-1}+(\delta-1) w_{2,3} v_{2}^{-1}-\delta w_{3} v_{3}^{-1}=0 \\
& w_{1,2} w_{1}^{-1}=v_{2} w_{2}^{-1} \\
& v_{2,3} w_{2}^{-1}=w_{3,2} w_{3}^{-1} \\
& v_{3} w_{3}^{-1}=v_{1,3} w_{1}^{-1} \\
& v_{1,2} v_{1}^{-1}=w_{2} v_{2}^{-1} \\
& w_{2,3} v_{2}^{-1}=v_{3,2} v_{3}^{-1} \\
& w_{3} v_{3}^{-1}=w_{1,3} v_{1}^{-1}
\end{aligned}
$$

Proposition 3.2. For any $w_{1}, v_{1}$ satisfying (3.6a) $-(3.6 \mathrm{~h})$,

$$
(w, v)=\left(w_{1}, v_{1}\right) \quad\left(l=n_{2}^{\prime}, m=n_{3}^{\prime}\right)
$$

solves $(3.2 \mathrm{a})$ and $(3.2 \mathrm{~b})$ with $a b=\delta$.

Proof. Let us rewrite (3.6a) using only $w_{1}, v_{1}$. From (3.6e) we immediately have

$$
w_{1,2} w_{1}^{-1}+(\delta-1) v_{2,3} w_{2}^{-1}-\delta v_{1,3} w_{1}^{-1}=0,
$$

and thus try to rewrite the second term. 
(3.6d) implies

$$
(\delta-1) v_{2,3} w_{2}^{-1}=(\delta-1) w_{3,2} w_{3}^{-1}=w_{3,2} v_{3,2}^{-1} \cdot(\delta-1) v_{3,2} v_{3}^{-1} \cdot v_{3} w_{3}^{-1} .
$$

Then, (3.6h) implies

$$
w_{3,2} v_{3,2}^{-1}=w_{1,23} v_{1,2}^{-1}, \quad v_{3} w_{3}^{-1}=v_{1} w_{1,3}^{-1},
$$

and (3.6g) implies

$$
(\delta-1) v_{3,2} v_{3}^{-1}=(\delta-1) w_{2,3} v_{2}^{-1} .
$$

By (3.6b) and (3.6h), this equation becomes

$$
(\delta-1) w_{2,3} v_{2}^{-1}=\delta w_{3} v_{3}^{-1}-v_{1,2} v_{1}^{-1}=\delta w_{1,3} v_{1}^{-1}-v_{1,2} v_{1}^{-1} .
$$

Combining (3.8)-(3.10), we obtain

$$
(\delta-1) v_{2,3} w_{2}^{-1}=w_{1,23} v_{1,2}^{-1} \cdot\left(\delta w_{1,3} v_{1}^{-1}-v_{1,2} v_{1}^{-1}\right) \cdot v_{1} w_{1,3}^{-1}=\delta w_{1,23} v_{1,2}^{-1}-w_{1,23} w_{1,3}^{-1} .
$$

Finally, (3.7) becomes

$$
w_{1,2} w_{1}^{-1}-w_{1,23} w_{1,3}^{-1}+\delta\left(w_{1,23} v_{1,2}^{-1}-v_{1,3} w_{1}^{-1}\right)=0 .
$$

In the same way, (3.6b) becomes

$$
v_{1,2} v_{1}^{-1}-v_{1,23} v_{1,3}^{-1}+\delta\left(v_{1,23} w_{1,2}^{-1}-w_{1,3} v_{1}^{-1}\right)=0 .
$$

If we set

$$
(w, v)=\left(w_{1}, v_{1}\right), \quad(l, m)=\left(n_{2}^{\prime}, n_{3}^{\prime}\right), \quad a b=\delta,
$$

we have

$$
w_{1,2}=w_{, l}, \quad w_{1,23}=w_{, l m}, \quad \text { etc. }
$$

Therefore, (3.11a) and (3.11b) become (3.2a) and (3.2b).

Remark 3.3. The solution constructed here seems to be only a part of the whole solutions of $(3.2 \mathrm{a})$ and $(3.2 \mathrm{~b})$, since it satisfies extra conditions

$$
w_{1,2} w_{1}^{-1} \cdot v_{1,2} v_{1}^{-1}=1, \quad v_{1,3} w_{1}^{-1} \cdot w_{1,3} v_{1}^{-1}=1 .
$$

\subsubsection{Continuum limit}

Assume $w$ is also a function $w(x, t)$ of continuum variables $x, t \in \mathbb{R}$ and has an expansion

$$
w(x+r, t+s)=w+\left(r w_{x}+s w_{t}\right)+\frac{1}{2}\left(r^{2} w_{x x}+2 r s w_{x t}+s^{2} w_{t t}\right)+\cdots,
$$

where $w_{x}=\partial w / \partial x$, etc. Connect $l, m$ to $x, t$ via the Miwa transformation

$$
w(x, t ; l, m)=w(x+l a, t+m b) .
$$

Assume similarly for $v=v(x, t ; l, m)$. Then we have

$$
w_{, l}=w+a w_{x}+\frac{a^{2}}{2} w_{x x}+\cdots,
$$




$$
\begin{aligned}
& w_{, l m}=w+\left(a w_{x}+b w_{t}\right)+\frac{1}{2}\left(a^{2} w_{x x}+2 a b w_{x t}+b^{2} w_{t t}\right)+\cdots, \\
& w_{, m}^{-1}=w^{-1}-b w^{-1} w_{t} w^{-1}-\frac{b^{2}}{2}\left(w^{-1} w_{t t} w^{-1}-2 w^{-1} w_{t} w^{-1} w_{t} w^{-1}\right)+\cdots, \\
& v_{, l}=\cdots,
\end{aligned}
$$

and from $(3.2 \mathrm{a}),(3.2 \mathrm{~b})$

$$
\begin{aligned}
0 & =w_{, l m} w_{, m}^{-1}-w_{, l} w^{-1}+a b\left(v_{, m} w^{-1}-w_{, l m} v_{, l}^{-1}\right) \\
& =a b\left(w_{x t} w^{-1}-w_{x} w^{-1} w_{t} w^{-1}+v w^{-1}-w v^{-1}\right)+(\text { higher-order terms }), \\
0 & =a b\left(v_{x t} v^{-1}-v_{x} v^{-1} v_{t} v^{-1}+w v^{-1}-v w^{-1}\right)+(\text { higher-order terms }) .
\end{aligned}
$$

Taking the limit $a, b \rightarrow 0$ successively, we obtain

$$
\begin{aligned}
& w_{x t} w^{-1}-w_{x} w^{-1} w_{t} w^{-1}+v w^{-1}-w v^{-1}=0, \\
& v_{x t} v^{-1}-v_{x} v^{-1} v_{t} v^{-1}+w v^{-1}-v w^{-1}=0 .
\end{aligned}
$$

Since $-w^{-1} w_{t} w^{-1}=\left(w^{-1}\right)_{t}$, these are transformed into

$$
\begin{aligned}
& \left(w_{x} w^{-1}\right)_{t}=w v^{-1}-v w^{-1}, \\
& \left(w_{x} w^{-1}+v_{x} v^{-1}\right)_{t}=0 .
\end{aligned}
$$

We call the pair (3.12a) and (3.12b) the noncommutative sine-Gordon equation. A quite similar equation with the same name has been derived in a different context [14, (3.10)].

Proposition 3.4. When $N=1$, the reduction condition

$$
w v=1
$$

gives the (commutative) sine-Gordon equation

$$
u_{x t}=4 \sin u,
$$

where $u$ is defined by

$$
u=\frac{2}{i} \log w .
$$

Proof. Under (3.13), (3.12b) clearly holds. And (3.14) is immediate from (3.12a) since

$$
u_{x t}=\frac{2}{i} \frac{w_{x t} w-w_{x} w_{t}}{w^{2}}, \quad \sin u=\frac{w^{2}-w^{-2}}{2 i} .
$$

\subsubsection{Darboux transformation}

When $(w, v)$ is a solution for (3.2a) and (3.2b), the column vector ${ }^{t}(\phi \psi)$ satisfying the linear system (3.1) is called the eigenfunction of $(w, v)$ for eigenvalue $\lambda$.

Let ${ }^{t}\left(\phi_{\lambda} \psi_{\lambda}\right),{ }^{t}\left(\phi_{\mu} \psi_{\mu}\right)$ be eigenfunctions of $(w, v)$ for eigenvalues $\lambda, \mu$, respectively. Define the Darboux transformation of $(w, v)$ and ${ }^{t}\left(\phi_{\lambda} \psi_{\lambda}\right)$ by ${ }^{t}\left(\phi_{\mu} \psi_{\mu}\right)$ as

$$
\widetilde{w}=\psi_{\mu} \phi_{\mu}^{-1} w, \quad \widetilde{v}=\phi_{\mu} \psi_{\mu}^{-1} v, \quad\left(\begin{array}{c}
\widetilde{\phi}_{\lambda} \\
\widetilde{\psi}_{\lambda}
\end{array}\right)=K\left(\begin{array}{c}
\phi_{\lambda} \\
\psi_{\lambda}
\end{array}\right)
$$

where

$$
K=\left(\begin{array}{cc}
-\mu \psi_{\mu} \phi_{\mu}^{-1} & \lambda \\
\lambda & -\mu \phi_{\mu} \psi_{\mu}^{-1}
\end{array}\right) .
$$


Theorem 3.5. $(\widetilde{w}, \widetilde{v})$ is a solution to $(3.2 \mathrm{a})$ and $(3.2 \mathrm{~b})$, and ${ }^{t}\left(\widetilde{\phi}_{\lambda} \widetilde{\psi}_{\lambda}\right)$ is an eigenfunction of $(\widetilde{w}, \widetilde{v})$ for eigenvalue $\lambda$.

Proof. From the linear system (3.1), we can write

$$
\begin{aligned}
& w_{, l} w^{-1}=\left(\phi_{\mu, l}+a \mu \psi_{\mu}\right) \phi_{\mu}^{-1}, \quad v_{, l} v^{-1}=\left(\psi_{\mu, l}+a \mu \phi_{\mu}\right) \psi_{\mu}^{-1}, \\
& w_{, m} v^{-1}=b^{-1} \mu\left(\phi_{\mu}-\phi_{\mu, m}\right) \psi_{\mu}^{-1}, \quad v_{, m} w^{-1}=b^{-1} \mu\left(\psi_{\mu}-\psi_{\mu, m}\right) \phi_{\mu}^{-1} .
\end{aligned}
$$

Then we have

$$
\begin{aligned}
& \widetilde{w}_{, l} \widetilde{w}^{-1}=\psi_{\mu, l}\left(\psi_{\mu}^{-1}+a \mu \phi_{\mu, l}^{-1}\right)=v_{, l} v^{-1}+a \mu\left(\psi_{\mu, l} \phi_{\mu, l}^{-1}-\phi_{\mu} \psi_{\mu}^{-1}\right), \\
& \widetilde{v}_{, l} \widetilde{v}^{-1}=\phi_{\mu, l}\left(\phi_{\mu}^{-1}+a \mu \psi_{\mu, l}\right)=w_{, l} w^{-1}+a \mu\left(\phi_{\mu, l} \psi_{\mu, l}^{-1}-\psi_{\mu} \phi_{\mu}^{-1}\right), \\
& \widetilde{w}_{, m} \widetilde{v}^{-1}=b^{-1} \mu \psi_{\mu, m}\left(\phi_{\mu, m}^{-1}-\phi_{\mu}^{-1}\right)=v_{m} w^{-1}+b^{-1} \mu\left(\psi_{\mu, m} \phi_{\mu, m}^{-1}-\psi_{\mu} \phi_{\mu}^{-1}\right), \\
& \widetilde{v}_{, m} \widetilde{w}^{-1}=b^{-1} \mu \phi_{\mu, m}\left(\psi_{\mu, m}^{-1}-\psi_{\mu}^{-1}\right)=w_{, m} v^{-1}+b^{-1} \mu\left(\phi_{\mu, m} \psi_{\mu, m}^{-1}-\phi_{\mu} \psi_{\mu}\right),
\end{aligned}
$$

which imply

$$
\begin{aligned}
& \left(\widetilde{w}_{, l} \widetilde{w}^{-1}\right)_{, m}-\widetilde{w}_{, l} \widetilde{w}^{-1}+a b\left(\widetilde{v}_{, m} \widetilde{w}^{-1}-\left(\widetilde{w}_{, m} \widetilde{v}^{-1}\right)_{, l}\right)=0, \\
& \left(\widetilde{v}_{, l} \widetilde{v}^{-1}\right)_{, m}-\widetilde{v}_{, l} \widetilde{v}^{-1}+a b\left(\widetilde{w}_{, m} \widetilde{v}^{-1}-\left(\widetilde{v}_{, m} \widetilde{w}^{-1}\right)_{, l}\right)=0 .
\end{aligned}
$$

Define $\widetilde{B}_{l}, \widetilde{B}_{m}$ by

$$
\widetilde{B}_{l}=\left(\begin{array}{cc}
\widetilde{w}_{, l} \widetilde{w}^{-1} & -a \lambda \\
-a \lambda & \widetilde{v}_{, l} \widetilde{v}^{-1}
\end{array}\right), \quad \widetilde{B}_{m}=\left(\begin{array}{cc}
1 & -b \lambda^{-1} \widetilde{w}_{, m} \widetilde{v}^{-1} \\
-b \lambda^{-1} \widetilde{v}_{, m} \widetilde{w}^{-1} & 1
\end{array}\right)
$$

Then we have

$$
K_{, l} B_{l}=\widetilde{B}_{l} K, \quad K_{, m} B_{m}=\widetilde{B}_{m} K
$$

and thus

$$
\begin{aligned}
& T_{l}\left(K\left(\begin{array}{l}
\phi \\
\psi
\end{array}\right)\right)=K_{, l} B_{l}\left(\begin{array}{l}
\phi \\
\psi
\end{array}\right)=\widetilde{B}_{l}\left(K\left(\begin{array}{l}
\phi \\
\psi
\end{array}\right)\right) \\
& T_{m}\left(K\left(\begin{array}{l}
\phi \\
\psi
\end{array}\right)\right)=K_{, m} B_{m}\left(\begin{array}{l}
\phi \\
\psi
\end{array}\right)=\widetilde{B}_{m}\left(K\left(\begin{array}{l}
\phi \\
\psi
\end{array}\right)\right) .
\end{aligned}
$$

\subsubsection{Multisoliton solutions}

The simplest solution for $(3.2 \mathrm{a})$ and $(3.2 \mathrm{~b})$ is the vacuum solution $(w, v)=(1,1)$. The linear system (3.1) of the vacuum solution is

$$
T_{l}\left(\begin{array}{l}
\phi \\
\psi
\end{array}\right)=\left(\begin{array}{cc}
1 & -a \lambda \\
-a \lambda & 1
\end{array}\right)\left(\begin{array}{l}
\phi \\
\psi
\end{array}\right), \quad T_{m}\left(\begin{array}{l}
\phi \\
\psi
\end{array}\right)=\left(\begin{array}{cc}
1 & -b \lambda^{-1} \\
-b \lambda^{-1} & 1
\end{array}\right)\left(\begin{array}{l}
\phi \\
\psi
\end{array}\right)
$$

which has two basic solutions

$$
\left(\begin{array}{l}
\phi \\
\psi
\end{array}\right)=\left(\begin{array}{c}
(1-a \lambda)^{l}\left(1-b \lambda^{-1}\right)^{m} \\
(1-a \lambda)^{l}\left(1-b \lambda^{-1}\right)^{m}
\end{array}\right),\left(\begin{array}{c}
(1+a \lambda)^{l}\left(1+b \lambda^{-1}\right)^{m} \\
-(1+a \lambda)^{l}\left(1+b \lambda^{-1}\right)^{m}
\end{array}\right) .
$$

Let $\lambda_{k}(k=1,2, \ldots)$ be mutually different eigenvalues and define

$$
\begin{aligned}
& \phi_{k}=\left(1-a \lambda_{k}\right)^{l}\left(1-b \lambda_{k}^{-1}\right)^{m}+\left(1+a \lambda_{k}\right)^{l}\left(1+b \lambda_{k}^{-1}\right)^{m} c_{k}, \\
& \psi_{k}=\left(1-a \lambda_{k}\right)^{l}\left(1-b \lambda_{k}^{-1}\right)^{m}-\left(1+a \lambda_{k}\right)^{l}\left(1+b \lambda_{k}^{-1}\right)^{m} c_{k},
\end{aligned}
$$



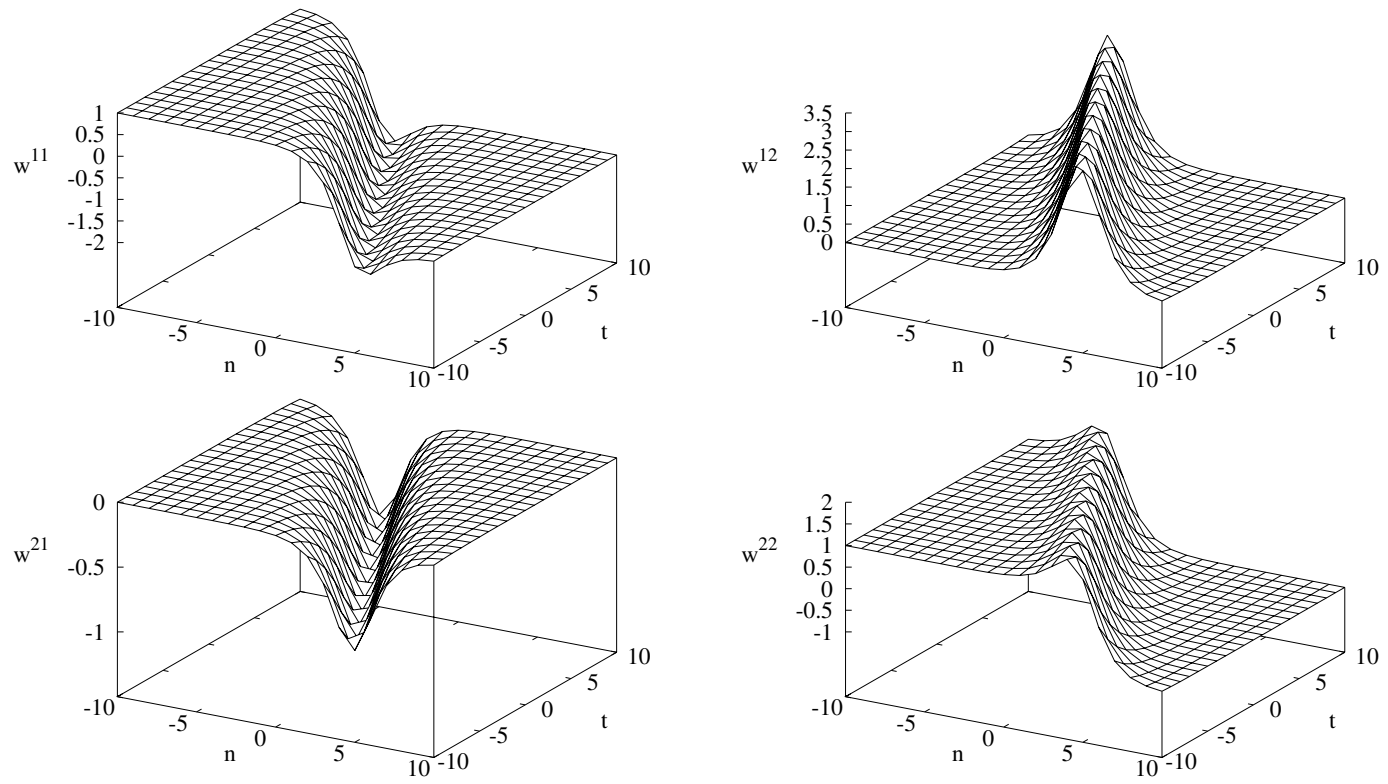

Figure 3.1. 1-soliton solution for ncdsG.

where $c_{k} \in \operatorname{Mat}(N, \mathbb{C})$ are parameters introducing noncommutativity. ${ }^{t}\left(\phi_{k} \psi_{k}\right)$ is of course an eigenfunction of the vacuum solution for eigenvalue $\lambda_{k}$. Repeating the Darboux transformation by ${ }^{t}\left(\phi_{k} \psi_{k}\right)$, we can construct multisoliton solutions.

A 1-soliton solution is given by

$$
\begin{aligned}
& w=\psi_{1} \phi_{1}^{-1}=\left(1-f_{1}\right)\left(1+f_{1}\right)^{-1}, \\
& v=\phi_{1} \psi_{1}^{-1}=\left(1+f_{1}\right)\left(1-f_{1}\right)^{-1},
\end{aligned}
$$

where $f_{k}$ is defined by

$$
f_{k}=\left(\frac{1+a \lambda_{k}}{1-a \lambda_{k}}\right)^{l}\left(\frac{1+b \lambda_{k}^{-1}}{1-b \lambda_{k}^{-1}}\right)^{m} c_{k} .
$$

As a concrete example, Fig. 3.1 shows the behavior of

$$
w=\left(\begin{array}{ll}
w^{11} & w^{12} \\
w^{21} & w^{22}
\end{array}\right) \quad(N=2)
$$

with

$$
a=b=0.2, \quad c_{1}=\left(\begin{array}{cc}
2 & -4 \\
1 & -1.5
\end{array}\right), \quad \lambda_{1}=\frac{5}{3}
$$

in the light-cone coordinates (2.11).

A 2 -soliton solution is given by

$$
\begin{aligned}
w= & \left(\lambda_{2} \phi_{2}-\lambda_{1} \phi_{1} \psi_{1}^{-1} \psi_{2}\right)\left(\lambda_{2} \psi_{2}-\lambda_{1} \psi_{1} \phi_{1}^{-1} \phi_{2}\right)^{-1} \psi_{1} \phi_{1}^{-1} \\
= & \left(\lambda_{2} \phi_{2} \psi_{2}^{-1}-\lambda_{1} \phi_{1} \psi_{1}^{-1}\right)\left(\lambda_{2} \phi_{1} \psi_{1}^{-1}-\lambda_{1} \phi_{2} \psi_{2}^{-1}\right)^{-1} \\
= & \left(\lambda_{2}\left(1+f_{2}\right)\left(1-f_{2}\right)^{-1}-\lambda_{1}\left(1+f_{1}\right)\left(1-f_{1}\right)^{-1}\right) \\
& \times\left(\lambda_{2}\left(1+f_{1}\right)\left(1-f_{1}\right)^{-1}-\lambda_{1}\left(1+f_{2}\right)\left(1-f_{2}\right)^{-1}\right)^{-1}, \\
v= & \left(\lambda_{2}\left(1-f_{2}\right)\left(1+f_{2}\right)^{-1}-\lambda_{1}\left(1-f_{1}\right)\left(1+f_{1}\right)^{-1}\right)
\end{aligned}
$$



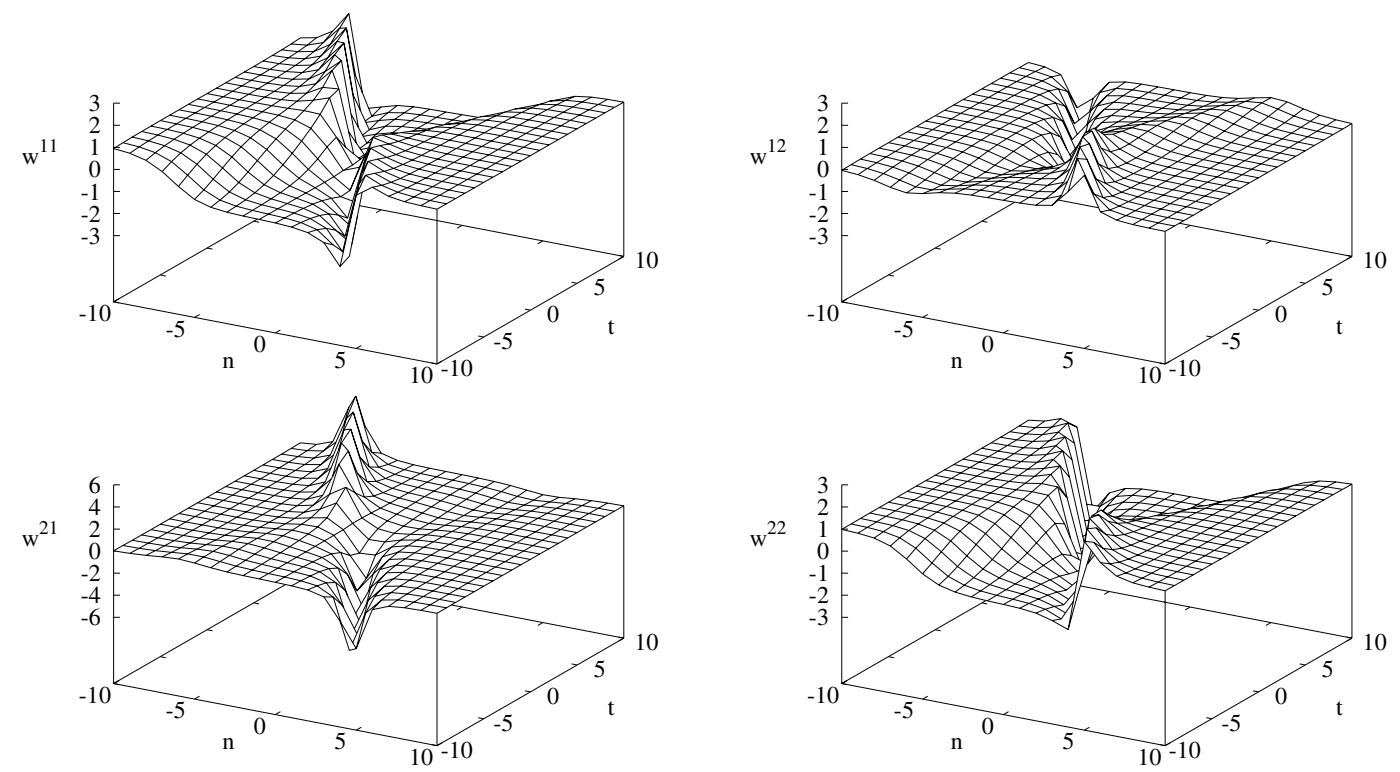

Figure 3.2. 2-soliton solution for ncdsG.

$$
\times\left(\lambda_{2}\left(1-f_{1}\right)\left(1+f_{1}\right)^{-1}-\lambda_{1}\left(1-f_{2}\right)\left(1+f_{2}\right)^{-1}\right)^{-1} .
$$

Fig. 3.2 shows the solution with

$$
a=b=0.2, \quad c_{1}=\left(\begin{array}{cc}
2.5 & -0.8 \\
2 & 1.8
\end{array}\right), \quad c_{2}=\left(\begin{array}{cc}
1.5 & 1.2 \\
-1 & 0.5
\end{array}\right), \quad \lambda_{1}=\lambda_{2}^{-1}=\frac{5}{3} .
$$

\subsubsection{Casoratian-type solutions}

Let $(w, v)$ be a solution for (3.2a) and $(3.2 \mathrm{~b}),{ }^{t}\left(\phi_{k} \psi_{k}\right)$ be eigenfunctions of $(w, v)$ for eigenvalues $\lambda_{k}(k=1,2, \ldots)$, where $\lambda_{k}$ are mutually different. Define repetition of the Darboux transformation by

$$
\begin{aligned}
& w^{(n)}=\psi_{n}^{(n)}\left(\phi_{n}^{(n)}\right)^{-1} w^{(n-1)}, \\
& v^{(n)}=\phi_{n}^{(n)}\left(\psi_{n}^{(n)}\right)^{-1} v^{(n-1)}, \\
& \phi_{k}^{(n+1)}=\lambda_{k} \psi_{k}^{(n)}-\lambda_{n} \psi_{n}^{(n)}\left(\phi_{n}^{(n)}\right)^{-1} \phi_{k}^{(n)}, \\
& \psi_{k}^{(n+1)}=\lambda_{k} \phi_{k}^{(n)}-\lambda_{n} \phi_{n}^{(n)}\left(\psi_{n}^{(n)}\right)^{-1} \psi_{k}^{(n)}
\end{aligned}
$$

and

$$
w^{(0)}=w, \quad v^{(0)}=v, \quad \phi_{k}^{(1)}=\phi_{k}, \quad \psi_{k}^{(1)}=\psi_{k} .
$$

For notational convenience, we introduce reduced shift operator $T$ defined by

$$
T f\left(\phi_{1}, \psi_{1}, \phi_{2}, \psi_{2}, \ldots\right)=f\left(\lambda_{1} \psi_{1}, \lambda_{1} \phi_{1}, \lambda_{2} \psi_{2}, \lambda_{2} \phi_{2}, \ldots\right),
$$

where $f\left(x_{1}, x_{2}, \ldots\right)$ is any rational function of noncommutative variables $x_{j}$. For example, we have

$$
T \phi_{k}=\lambda_{k} \psi_{k}, \quad T \psi_{k}=\lambda_{k} \phi_{k}, \quad T^{2} \phi_{k}=\lambda_{k}^{2} \phi_{k} .
$$




\section{Lemma 3.6.}

$$
T \phi_{k}^{(n+1)}=\lambda_{k} \psi_{k}^{(n+1)}, \quad T \psi_{k}^{(n+1)}=\lambda_{k} \phi_{k}^{(n+1)} .
$$

Proof. We prove by induction. Obviously, $\phi_{k}^{(n+1)}, \psi_{k}^{(n+1)}$ are rational functions of $\phi_{j}, \psi_{j}$. Assume $T \phi_{k}^{(n)}=\lambda_{k} \psi_{k}^{(n)}, T \psi_{k}^{(n)}=\lambda_{k} \phi_{k}^{(n)}$ for certain $n$. Then,

$$
T \phi_{k}^{(n+1)}=\lambda_{k}\left(\lambda_{k} \phi_{k}^{(n)}\right)-\lambda_{n}\left(\lambda_{n} \phi_{n}^{(n)}\right)\left(\lambda_{n} \psi_{n}^{(n)}\right)^{-1} \lambda_{k} \psi_{k}^{(n)}=\lambda_{k} \psi_{k}^{(n+1)} .
$$

Similarly, $T \psi_{k}^{(n+1)}=\lambda_{k} \phi_{k}^{(n+1)}$.

\section{Theorem 3.7.}

$$
\begin{aligned}
& w^{(n)}=\prod_{j=1}^{n}\left(-\lambda_{j}^{-1}\right) \cdot\left|\begin{array}{ccccc}
\phi_{1} & \phi_{2} & \cdots & \phi_{n} & 1 \\
T \phi_{1} & T \phi_{2} & \cdots & T \phi_{n} & 0 \\
\vdots & \vdots & \ddots & \vdots & \vdots \\
T^{n-1} \phi_{1} & T^{n-1} \phi_{2} & \cdots & T^{n-1} \phi_{n} & 0 \\
T^{n} \phi_{1} & T^{n} \phi_{2} & \cdots & T^{n} \phi_{n} & 0
\end{array}\right| w, \\
& v^{(n)}=\prod_{j=1}^{n}\left(-\lambda_{j}^{-1}\right) \cdot\left|\begin{array}{ccccc}
\psi_{1} & \psi_{2} & \cdots & \psi_{n} & 1 \\
T \psi_{1} & T \psi_{2} & \cdots & T \psi_{n} & 0 \\
\vdots & \vdots & \ddots & \vdots & \vdots \\
T^{n-1} \psi_{1} & T^{n-1} \psi_{2} & \cdots & T^{n-1} \psi_{n} & 0 \\
T^{n} \psi_{1} & T^{n} \psi_{2} & \cdots & T^{n} \psi_{n} & 0
\end{array}\right| v, \\
& \phi_{k}^{(n+1)}=\left|\begin{array}{ccccc}
\phi_{1} & \phi_{2} & \cdots & \phi_{n} & \phi_{k} \\
T \phi_{1} & T \phi_{2} & \cdots & T \phi_{n} & T \phi_{k} \\
\vdots & \vdots & \ddots & \vdots & \vdots \\
T^{n-1} \phi_{1} & T^{n-1} \phi_{2} & \cdots & T^{n-1} \phi_{n} & T^{n-1} \phi_{k} \\
T^{n} \phi_{1} & T^{n} \phi_{2} & \cdots & T^{n} \phi_{n} & T^{n} \phi_{k}
\end{array}\right|, \\
& \psi_{k}^{(n+1)}=\left|\begin{array}{ccccc}
\psi_{1} & \psi_{2} & \cdots & \psi_{n} & \psi_{k} \\
T \psi_{1} & T \psi_{2} & \cdots & T \psi_{n} & T \psi_{k} \\
\vdots & \vdots & \ddots & \vdots & \vdots \\
T^{n-1} \psi_{1} & T^{n-1} \psi_{2} & \cdots & T^{n-1} \psi_{n} & T^{n-1} \psi_{k} \\
T^{n} \psi_{1} & T^{n} \psi_{2} & \cdots & T^{n} \psi_{n} & T^{n} \psi_{k}
\end{array}\right| .
\end{aligned}
$$

Here, quasideterminants [5] are used (see Appendix B). When $n=0,(3.17 \mathrm{a})$ and (3.17b) read

$$
w^{(0)}=1 \cdot\left\|1\left|w, \quad v^{(0)}=1 \cdot \| 1\right| v,\right.
$$

respectively.

Before proceeding to the proof, we prepare the following lemma.

Lemma 3.8. Let $C=\left(c_{i j}\right), C^{\prime}=\left(c_{i j}^{\prime}\right)$ be $n \times n$ matrices where $c_{i j}, c_{i j}^{\prime} \in \operatorname{Mat}(N, \mathbb{C})$. Assume $c_{i j}=c_{i j}^{\prime}$ for $1 \leq i \leq n, 1 \leq j \leq n-1$. Then,

$$
|C|_{p_{1} n}^{-1}\left|C^{\prime}\right|_{p_{1} n}=|C|_{p_{2} n}^{-1}\left|C^{\prime}\right|_{p_{2} n} .
$$

Proof. By the column homological relation (Proposition B.3), we have

$$
\begin{aligned}
& \left|C^{p_{2} n}\right|_{p_{1} j}^{-1}|C|_{p_{1} n}=-\left|C^{p_{1} n}\right|_{p_{2} j}|C|_{p_{2} n}, \\
& \left|C^{\prime p_{2} n}\right|_{p_{1} j}^{-1}\left|C^{\prime}\right|_{p_{1} n}=-\left|C^{\prime p_{1} n}\right|_{p_{2} j}\left|C^{\prime}\right|_{p_{2} n} .
\end{aligned}
$$

By the assumption, we have $C^{p_{2} n}=C^{\prime p_{2} n}, C^{p_{1} n}=C^{p_{1} n}$. Therefore, we obtain (3.18) by multiplying the inverse of (3.19a) to (3.19b) from the left. 
Proof of Theorem 3.7. We prove by induction. The case $n=0$ is trivial.

Assume $w^{(n-1)}, v^{(n-1)}, \phi_{k}^{(n)}, \psi_{k}^{(n)}$ have the above expressions for certain $n>0$. Then,

$$
\begin{aligned}
w^{(n)}= & \lambda_{n}^{-1}\left(T \phi_{n}^{(n)}\right)\left(\phi_{n}^{(n)}\right)^{-1} w^{(n-1)} \\
= & -\prod_{j=1}^{n}\left(-\lambda_{j}^{-1}\right) \cdot\left|\begin{array}{ccc}
T \phi_{1} & \cdots & T \phi_{n} \\
\vdots & \ddots & \vdots \\
T^{n} \phi_{1} & \cdots & T^{n} \phi_{n}
\end{array}\right|\left|\begin{array}{ccc}
\phi_{1} & \cdots & \phi_{n} \\
\vdots & \ddots & \vdots \\
T^{n-1} \phi_{1} & \cdots & T^{n-1} \phi_{n}
\end{array}\right| \\
& \times\left|\begin{array}{cccc}
\phi_{1} & \cdots & \phi_{n-1} & 1 \\
T \phi_{1} & \cdots & T \phi_{n-1} & 0 \\
\vdots & \ddots & \vdots & \vdots \\
T^{n-1} \phi_{1} & \cdots & T^{n-1} \phi_{n-1} & 0
\end{array}\right| w .
\end{aligned}
$$

By Lemma 3.8, we obtain

$$
\begin{aligned}
& \left|\begin{array}{ccc}
\phi_{1} & \cdots & \phi_{n} \\
\vdots & \ddots & \vdots \\
T^{n-1} \phi_{1} & \cdots & T^{n-1} \phi_{n}
\end{array}\right|^{-1}\left|\begin{array}{cccc}
\phi_{1} & \cdots & \phi_{n-1} & 1 \\
T \phi_{1} & \cdots & T \phi_{n-1} & 0 \\
\vdots & \ddots & \vdots & \vdots \\
T^{n-1} \phi_{1} & \cdots & T^{n-1} \phi_{n-1} & 0
\end{array}\right| \\
& =\left|\begin{array}{ccc}
\phi_{1} & \cdots & \phi_{n} \\
\vdots & \ddots & \vdots \\
T^{n-1} \phi_{1} & \cdots & T^{n-1} \phi_{n}
\end{array}\right|^{-1}\left|\begin{array}{cccc}
\phi_{1} & \cdots & \phi_{n-1} & 1 \\
T \phi_{1} & \cdots & T \phi_{n-1} & 0 \\
\vdots & \ddots & \vdots & \vdots \\
T^{n-1} \phi_{1} & \cdots & T^{n-1} \phi_{n-1} & 0
\end{array}\right| .
\end{aligned}
$$

We define an $(n-1) \times(n-1)$ matrix $A_{0}$ by

$$
A_{0}=\left(\begin{array}{ccc}
T \phi_{1} & \cdots & T \phi_{n-1} \\
\vdots & \ddots & \vdots \\
T^{n-1} \phi_{1} & \cdots & T^{n-1} \phi_{n-1}
\end{array}\right)
$$

With $A_{0}$, the above quasideterminants are rewritten as

$$
\begin{aligned}
& \left|\begin{array}{ccc}
T \phi_{1} & \cdots & T \phi_{n} \\
\vdots & \ddots & \vdots \\
T^{n} \phi_{1} & \cdots & T^{n} \phi_{n}
\end{array}\right|=\left|\begin{array}{cccc} 
& & & T \phi_{n} \\
& A_{0} & & \vdots \\
& & & T^{n-1} \phi_{n} \\
T^{n} \phi_{1} & \cdots & T^{n} \phi_{n-1} & T^{n} \phi_{n}
\end{array}\right|, \\
& \left|\begin{array}{ccc}
\phi_{1} & \cdots & \phi_{n} \\
\vdots & \ddots & \vdots \\
T^{n-1} \phi_{1} & \cdots & T^{n-1} \phi_{n}
\end{array}\right|=\left|\begin{array}{cccc}
\phi_{1} & \cdots & \phi_{n-1} & \frac{\phi_{n}}{T \phi_{n}} \\
& & & \vdots \\
& A_{0} & & T^{n-1} \phi_{n}
\end{array}\right|, \\
& \left|\begin{array}{cccc}
\phi_{1} & \cdots & \phi_{n-1} & 1 \\
T \phi_{1} & \cdots & T \phi_{n-1} & 0 \\
\vdots & \ddots & \vdots & \vdots \\
T^{n-1} \phi_{1} & \cdots & T^{n-1} \phi_{n-1} & 0
\end{array}\right|=\left|\begin{array}{cccc}
\phi_{1} & \cdots & \phi_{n-1} & 1 \\
& & & 0 \\
& A_{0} & & \vdots \\
& & & 0
\end{array}\right|,
\end{aligned}
$$


and also, we have a trivial identity

$$
0=\left|\begin{array}{cccc} 
& & & 0 \\
& A_{0} & & \vdots \\
& & & 0 \\
T^{n} \phi_{1} & \cdots & T^{n} \phi_{n-1} & 0
\end{array}\right| .
$$

By the invariance under row and column permutations (Proposition B.2) and Sylvester's identity (Proposition B.4), we can combine these four quasideterminants into one to obtain

$$
w^{(n)}=\prod_{j=1}^{n}\left(-\lambda_{j}^{-1}\right) \cdot\left|\begin{array}{ccccc}
\phi_{1} & \cdots & \phi_{n-1} & \phi_{n} & 1 \\
& & & T \phi_{n} & 0 \\
& A_{0} & & \vdots & \vdots \\
& & & T^{n-1} \phi_{n} & 0 \\
T^{n} \phi_{1} & \cdots & T^{n} \phi_{n-1} & T^{n} \phi_{n} & 0
\end{array}\right| w .
$$

Similarly for $v^{(n)}$.

For $\phi_{k}^{(n+1)}$, we have

$$
\begin{aligned}
& \phi_{k}^{(n+1)}=T \phi_{k}^{(n)}-\left(T \phi_{n}^{(n)}\right)\left(\phi_{n}^{(n)}\right)^{-1} \phi_{k}^{(n)} \\
& =\left|\begin{array}{cccc}
T \phi_{1} & \cdots & T \phi_{n-1} & T \phi_{k} \\
\vdots & \ddots & \vdots & \vdots \\
T^{n} \phi_{1} & \cdots & T^{n} \phi_{n-1} & T^{n} \phi_{k}
\end{array}\right|-\left|\begin{array}{cccc}
T \phi_{1} & \cdots & T \phi_{n-1} & T \phi_{n} \\
\vdots & \ddots & \vdots & \vdots \\
T^{n} \phi_{1} & \cdots & T^{n} \phi_{n-1} & T^{n} \phi_{n}
\end{array}\right| \\
& \times\left|\begin{array}{cccc}
\phi_{1} & \cdots & \phi_{n-1} & \phi_{n} \\
T \phi_{1} & \cdots & T \phi_{n-1} & T \phi_{n} \\
\vdots & \ddots & \vdots & \vdots \\
T^{n-1} \phi_{1} & \cdots & T^{n-1} \phi_{n-1} & T^{n-1} \phi_{n}
\end{array}\right|^{-1} \\
& \times\left|\begin{array}{cccc}
\phi_{1} & \cdots & \phi_{n-1} & \phi_{k} \\
T \phi_{1} & \cdots & T \phi_{n-1} & T \phi_{k} \\
\vdots & \ddots & \vdots & \vdots \\
T^{n-1} \phi_{1} & \cdots & T^{n-1} \phi_{n-1} & T^{n-1} \phi_{k}
\end{array}\right| .
\end{aligned}
$$

With the same technique for $w^{(n)}$, we obtain

$$
\phi_{k}^{(n+1)}=\left|\begin{array}{cccc}
\phi_{1} & \cdots & \phi_{n} & \phi_{k} \\
\vdots & \ddots & \vdots & \vdots \\
T^{n} \phi_{1} & \cdots & T^{n} \phi_{n} & T^{n} \phi_{k}
\end{array}\right| .
$$

Similarly for $\psi_{k}^{(n+1)}$.

\subsection{Noncommutative ultradiscrete sine-Gordon equation}

\subsubsection{Ultradiscretization}

We perform ultradiscretization of ncdsG by the parametrization

$$
a=\mu_{A} e^{\widetilde{A} s}, \quad b=\mu_{B} e^{\widetilde{A} s}, \quad \widetilde{A}, \widetilde{B}<0 .
$$


Assuming

$$
a \stackrel{\mathrm{ud}}{\longrightarrow} A, \quad b \stackrel{\mathrm{ud}}{\longrightarrow} B, \quad w \stackrel{\mathrm{ud}}{\longrightarrow} W, \quad v \stackrel{\mathrm{ud}}{\longrightarrow} V,
$$

we obtain

$$
\begin{aligned}
& W_{, l m} W_{, m}^{-1} \ominus W_{, l} W^{-1} \oplus A B\left(V_{, m} W^{-1} \ominus W_{, l m} V_{, l}^{-1}\right) \nabla-\infty, \\
& V_{, l m} V_{, m}^{-1} \ominus V_{, l} V^{-1} \oplus A B\left(W_{, m} V^{-1} \ominus V_{, l m} W_{, l}^{-1}\right) \nabla-\infty .
\end{aligned}
$$

We call the pair (3.20a) and (3.20b) the noncommutative ultradiscrete sine-Gordon equation (ncudsG). Because $u \operatorname{Mat}(N, \mathrm{u} \mathbb{C})$ can be realized by $\operatorname{uMat}(2 N, \mathrm{u} \mathbb{R})$, we use $\operatorname{uMat}(N, u \mathbb{R})$ as the underlying algebra for simplicity.

\subsubsection{1-soliton solution}

In order to ultradiscretize solutions for ncdsG, we introduce

$$
p_{j}=\frac{1+a \lambda_{j}}{1-a \lambda_{j}}, \quad q_{j}=\frac{1+b \lambda_{j}^{-1}}{1-b \lambda_{j}^{-1}} .
$$

These solve the dispersion relation

$$
(1-a b)\left(1+p_{j} q_{j}\right)=(1+a b)\left(p_{j}+q_{j}\right),
$$

and any solution of (3.22) is parametrized by $\lambda_{j}$ through (3.21) unless $a b=1$. As in the commutative case, (3.22) is ultradiscretized to

$$
0 \oplus P_{j} Q_{j} \nabla P_{j} \oplus Q_{j},
$$

where $p_{j} \stackrel{\mathrm{ud}}{\longrightarrow} P_{j}, q_{j} \stackrel{\mathrm{ud}}{\longrightarrow} Q_{j}$.

We can directly discretize the 1-soliton solution (3.15a), (3.15b) to obtain

$$
w \stackrel{\mathrm{ud}}{\longrightarrow} W \nabla\left(0 \ominus F_{1}\right)\left(0 \oplus F_{1}\right)^{-1}, \quad v \stackrel{\mathrm{ud}}{\longrightarrow} V \nabla\left(0 \oplus F_{1}\right)\left(0 \ominus F_{1}\right)^{-1},
$$

where

$$
F_{j}=P_{j}^{l} Q_{j}^{m} C_{j}, \quad c_{j} \stackrel{\mathrm{ud}}{\longrightarrow} C_{j} \in \operatorname{uMat}(N, \mathrm{u} \mathbb{R}) .
$$

This relation is valid, but inadequate to determine $W, V$ in many cases. For simplicity, we assume $N=2$ hereafter. If we write $W=\left(W^{\iota \kappa}\right), F_{j}=\left(F_{j}^{\iota \kappa}\right)$, the $(1,2)$-th element of $(0 \ominus$ $\left.F_{1}\right)\left(0 \oplus F_{1}\right)^{-1}$ is given by

$$
\frac{\ominus F_{1}^{12}\left(\left(0 \ominus F_{1}^{11}\right) \oplus\left(0 \oplus F_{1}^{11}\right)\right)}{\operatorname{det}\left(0 \oplus F_{1}\right)}=\frac{\ominus F_{1}^{12}\left(0 \oplus\left(F_{1}^{11}\right)^{\bullet}\right)}{\operatorname{det}\left(0 \oplus F_{1}\right)}
$$

and $\left|F_{1}^{11}\right|_{\oplus}$ exceeds 0 for large $\pm l$ or $\pm m$. Then this element is balanced and $W^{12}$ cannot be determined. Therefore, we need more precise expressions to ultradiscretize.

Define

$$
g_{j}=\left(1+f_{j}\right)\left(1-f_{j}\right)^{-1}, \quad h_{j}=\left(1-f_{j}\right)\left(1+f_{j}\right)^{-1} .
$$


Of course, $w=h_{1}, v=g_{1}$ is a 1-soliton solution for ncdsG. Writing $f_{j}=\left(f_{j}^{\iota \kappa}\right)$, we have

$$
\begin{aligned}
& g_{j}=\left(\begin{array}{cc}
\frac{\left(1+f_{j}^{11}\right)\left(1-f_{j}^{22}\right)+f_{j}^{12} f_{j}^{21}}{\operatorname{det}\left(1-f_{j}\right)} & \frac{2 f_{j}^{12}}{\operatorname{det}\left(1-f_{j}\right)} \\
\frac{2 f_{j}^{21}}{\operatorname{det}\left(1-f_{j}\right)} & \frac{\left(1+f_{j}^{22}\right)\left(1-f_{j}^{11}\right)+f_{j}^{21} f_{j}^{12}}{\operatorname{det}\left(1-f_{j}\right)}
\end{array}\right), \\
& h_{j}=\left(\begin{array}{cc}
\frac{\left(1-f_{j}^{11}\right)\left(1+f_{j}^{22}\right)+f_{j}^{12} f_{j}^{21}}{\operatorname{det}\left(1+f_{j}\right)} & \frac{-2 f_{j}^{12}}{\operatorname{det}\left(1+f_{j}\right)} \\
\frac{-2 f_{j}^{21}}{\operatorname{det}\left(1+f_{j}\right)} & \frac{\left(1-f_{j}^{22}\right)\left(1+f_{j}^{11}\right)+f_{j}^{21} f_{j}^{12}}{\operatorname{det}\left(1+f_{j}\right)}
\end{array}\right) .
\end{aligned}
$$

By ultradiscretization, we obtain

$$
\begin{array}{r}
g_{j} \stackrel{\mathrm{ud}}{\longrightarrow} G_{j} \nabla\left(\begin{array}{cc}
\frac{\left(0 \oplus F_{j}^{11}\right)\left(0 \ominus F_{j}^{22}\right) \oplus F_{j}^{12} F_{j}^{21}}{\operatorname{det}\left(0 \ominus F_{j}\right)} & \frac{F_{j}^{12}}{\operatorname{det}\left(0 \ominus F_{j}\right)} \\
\frac{F_{j}^{21}}{\operatorname{det}\left(0 \ominus F_{j}\right)} & \frac{\left(0 \oplus F_{j}^{22}\right)\left(0 \ominus F_{j}^{11}\right) \oplus F_{j}^{21} F_{j}^{12}}{\operatorname{det}\left(0 \ominus F_{j}\right)}
\end{array}\right), \\
h_{j} \stackrel{\mathrm{ud}}{\longrightarrow} H_{j} \nabla\left(\begin{array}{cc}
\frac{\left(0 \ominus F_{j}^{11}\right)\left(0 \oplus F_{j}^{22}\right) \oplus F_{j}^{12} F_{j}^{21}}{\operatorname{det}\left(0 \oplus F_{j}\right)} & \frac{\ominus F_{j}^{12}}{\operatorname{det}\left(0 \oplus F_{j}\right)} \\
\frac{\ominus F_{j}^{21}}{\operatorname{det}\left(0 \oplus F_{j}\right)} & \frac{\left(0 \ominus F_{j}^{22}\right)\left(0 \oplus F_{j}^{11}\right) \oplus F_{j}^{21} F_{j}^{12}}{\operatorname{det}\left(0 \oplus F_{j}\right)}
\end{array}\right) .
\end{array}
$$

We can choose $C_{j}, P_{j}, Q_{j} \in \mathrm{u} \mathbb{Z}$ such that $\left(0 \oplus F_{j}^{11}\right)\left(0 \ominus F_{j}^{22}\right)$ is always even and $F_{j}^{12} F_{j}^{21}$ odd. Then all the elements on the r.h.s. of (3.24a), (3.24b) are signed and $G_{j}, H_{j}$ are completely determined. Fig. 3.3 shows $W=H_{1}$ with

$$
A=B=-1, \quad C_{1}=\left(\begin{array}{cc}
\ominus(-7) & -8 \\
\ominus(-5) & \ominus 7
\end{array}\right), \quad P_{1}=2, \quad Q_{1}=0 .
$$

\subsubsection{2-soliton solution}

Ultradiscretization of (3.16a), (3.16b) gives

$$
\begin{aligned}
& W \nabla\left(L_{2} G_{2} \ominus L_{1} G_{1}\right)\left(L_{2} G_{1} \ominus L_{1} G_{2}\right)^{-1}, \\
& V \nabla\left(L_{2} H_{2} \ominus L_{1} H_{1}\right)\left(L_{2} H_{1} \ominus L_{1} H_{2}\right)^{-1},
\end{aligned}
$$

where

$$
\lambda_{j} \stackrel{\mathrm{ud}}{\longrightarrow} L_{j} .
$$

In order to determine the value of $L_{j}$, we examine the relation

$$
\lambda_{j}=\frac{p_{j}-1}{a\left(p_{j}+1\right)}=\frac{b\left(q_{j}+1\right)}{q_{j}-1} .
$$



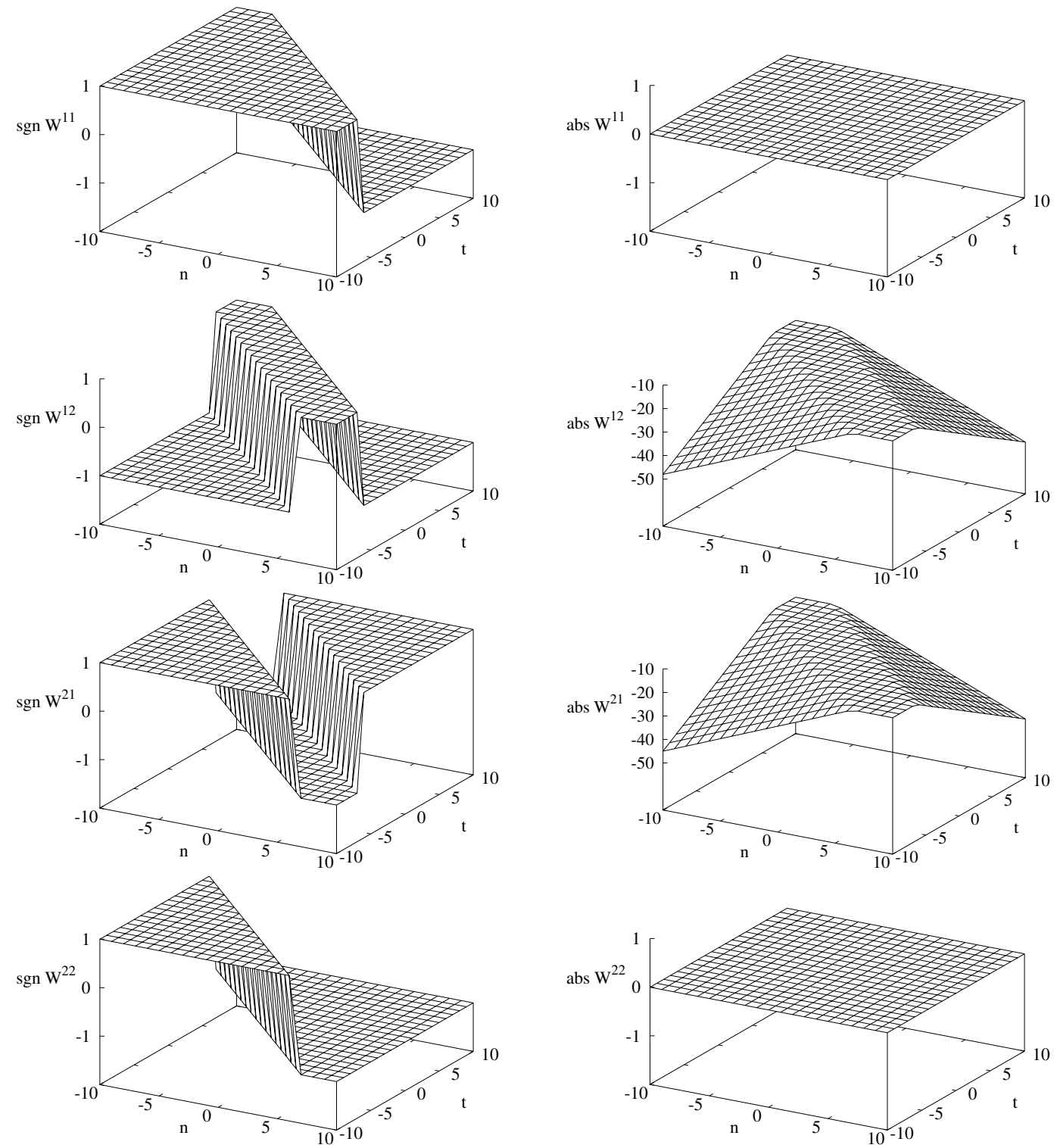

Figure 3.3. 1-soliton solution for ncudsG.

By the dispersion relation (3.23), we have $P_{j}=0$ or $Q_{j}=0$. When $Q_{j}=0, q_{j}$ behaves like a constant with regard to the ultradiscretization parameter $s$ and $p_{j}$ cannot behave like one. Therefore, we have

$$
L_{j}=\frac{P_{j} \ominus 0}{A\left(P_{j} \oplus 0\right)}= \begin{cases}A^{-1}, & \left|P_{j}\right|_{\oplus}>0 \\ \ominus A^{-1}, & \left|P_{j}\right|_{\oplus}<0 .\end{cases}
$$

Similarly, when $P_{j}=0$, we have

$$
L_{j}=\frac{B\left(Q_{j} \oplus 0\right)}{Q_{j} \ominus 0}= \begin{cases}B, & \left|Q_{j}\right|_{\oplus}>0, \\ \ominus B, & \left|Q_{j}\right|_{\oplus}<0 .\end{cases}
$$

If we choose $P_{2}=Q_{1}=0$, we have $\left|L_{1}\right|_{\oplus}>\left|L_{2}\right|_{\oplus}$ and thus

$$
W \nabla G_{1} G_{2}^{-1}, \quad V \nabla H_{1} H_{2}^{-1} \text {. }
$$



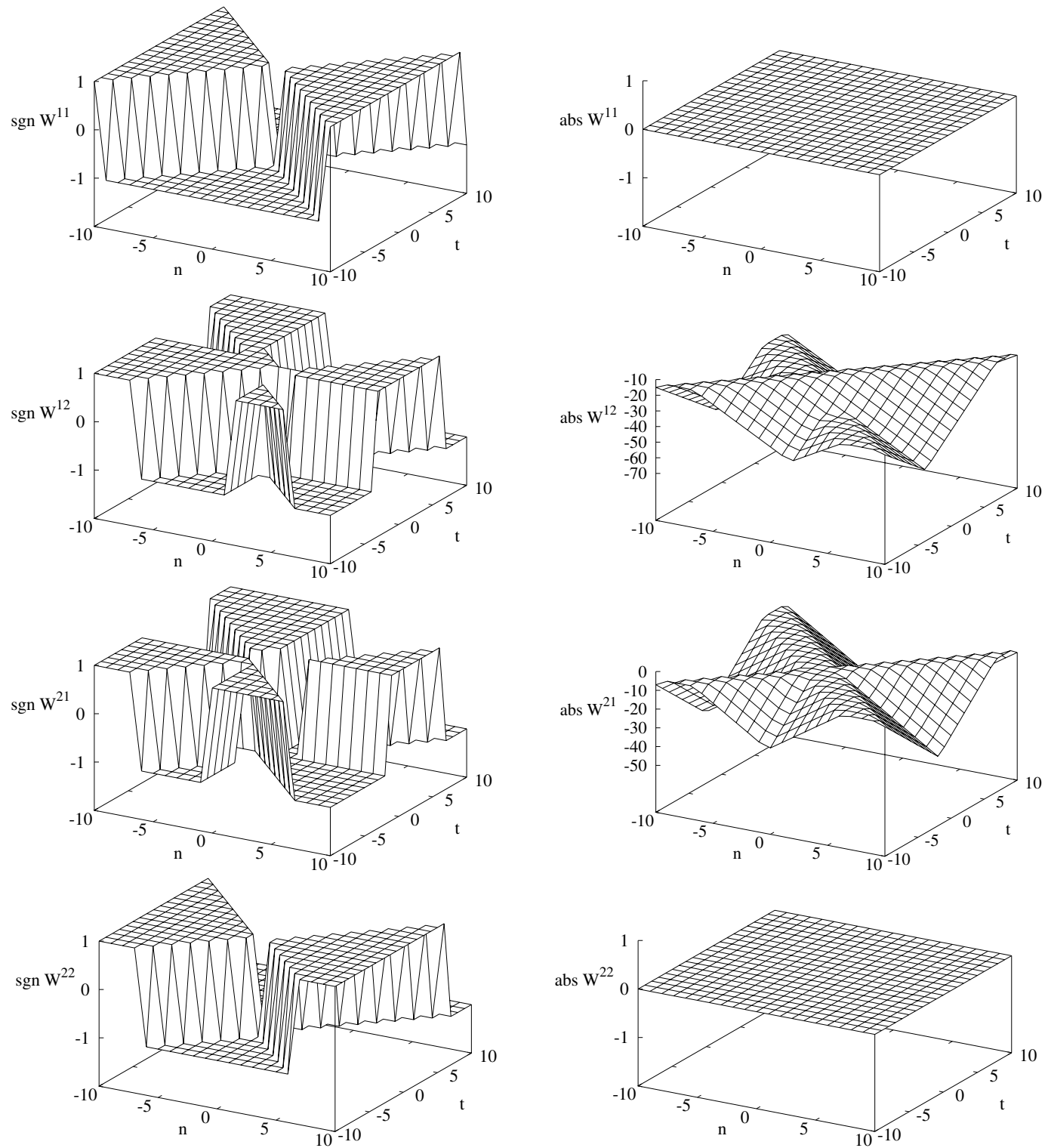

Figure 3.4. 2-soliton solution for ncudsG.

Similarly, if $P_{1}=Q_{2}=0$,

$$
W \nabla G_{2} G_{1}^{-1}, \quad V \nabla H_{2} H_{1}^{-1} \text {. }
$$

Fig. 3.4 shows behavior of $W=G_{1} G_{2}^{-1}$ with parameters $A=B=-1$,

$$
C_{1}=\left(\begin{array}{cc}
\ominus 2 & \ominus(-13) \\
\ominus 11 & 15
\end{array}\right), \quad C_{2}=\left(\begin{array}{cc}
-3 & \ominus(-15) \\
\ominus(-7) & \ominus(-13)
\end{array}\right), \quad P_{1}=Q_{2}=4
$$

These are chosen so that every elements involved are signed.

\section{Conclusion and discussion}

We have proposed an ultradiscrete analogue of the sine-Gordon equation and constructed signed 1-soliton and 2-soliton solutions utilizing $u \mathbb{R}$. The traveling-wave, kink-antikink, and kink-kink 
solutions, which contain ultradiscrete complex numbers, do exist and their correspondence to those for the discrete sine-Gordon equation is quite clear. When the range of solutions are restricted to $u \mathbb{R}$, even deterministic time evolution is possible.

As stated in Section 1, another ultradiscretization of the sine-Gordon equation has been given by Isojima et al. [9, 10]. There, only $\tau$ is ultradiscretized, no complex solutions are dealt with, and time evolution is not possible. Our formulation looks better in these respects. Also, ultradiscretization via the parametrization (2.16) can be considered as another aspect of continuum limit, but ultradiscretization in [9] cannot since they choose a parametrization such that $\delta \rightarrow \pm 1$.

We have also proposed a noncommutative discrete analogue of the sine-Gordon equation and revealed its relation to other integrable systems including the noncommutative discrete KP equation. Also, multisoliton solutions are constructed by a repeated application of Darboux transformations. And finally, a noncommutative ultradiscrete analogue of the sine-Gordon equation and its signed 1-soliton and 2-soliton solutions are derived by ultradiscretization with $u \mathbb{R}$.

\section{A Symmetrized max-plus algebra and ultradiscretization}

We make extensive use of the symmetrized max-plus algebra $u \mathbb{R}$ in the main part of the paper. Therefore we describe basic definitions and properties of uR here. For details, see Baccelli et al. [2].

\section{A.1 Symmetrized max-plus algebra}

\section{A.1.1 Pair of the max-plus algebra}

Let $\mathbb{R}_{\max }=\mathbb{R} \cup\{-\infty\} . \mathbb{R}_{\max }$ has the obvious total order. Define $\oplus$ and $\otimes$ by

$$
x \oplus y=\max (x, y), \quad x \otimes y=x+y
$$

for $x, y \in \mathbb{R}_{\max }$. With these operations, $\mathbb{R}_{\max }$ becomes a commutative dioid called the max-plus algebra. The null element is $-\infty$ and the unit element is 0 . We extend $\oplus$ and $\otimes$ over $\mathbb{R}_{\max }^{2}$ by

$$
\begin{aligned}
& \left(x_{1}, x_{2}\right) \oplus\left(y_{1}, y_{2}\right)=\left(x_{1} \oplus y_{1}, x_{2} \oplus y_{2}\right), \\
& \left(x_{1}, x_{2}\right) \otimes\left(y_{1}, y_{2}\right)=\left(x_{1} y_{1} \oplus x_{2} y_{2}, x_{1} y_{2} \oplus x_{2} y_{1}\right) .
\end{aligned}
$$

Then $\mathbb{R}_{\max }^{2}$ is a commutative dioid with null element $(-\infty,-\infty)$ and unit element $(0,-\infty)$. $\mathbb{R}_{\max }$ is embedded into $\mathbb{R}_{\max }^{2}$ by $x \longmapsto(x,-\infty)$.

Define minus sign $\ominus$ by

$$
\ominus\left(x_{1}, x_{2}\right)=\left(x_{2}, x_{1}\right)
$$

for $x=\left(x_{1}, x_{2}\right) \in \mathbb{R}_{\max }^{2}$. We write $x \ominus y$ for $x \oplus(\ominus y)$, which is regarded as subtraction. Define absolute value $\|_{\oplus}: \mathbb{R}_{\max }^{2} \rightarrow \mathbb{R}_{\max }$ by

$$
\left|\left(x_{1}, x_{2}\right)\right|_{\oplus}=x_{1} \oplus x_{2}
$$

Define balance operator ${ }^{\bullet}$ by

$$
\left(x_{1}, x_{2}\right)^{\bullet}=\left(x_{1}, x_{2}\right) \ominus\left(x_{1}, x_{2}\right)=\left(x_{1} \oplus x_{2}, x_{1} \oplus x_{2}\right) .
$$




\section{A.1.2 Symmetrized max-plus algebra}

It is natural to consider the balance relation $\nabla$ defined by

$$
\left(x_{1}, x_{2}\right) \nabla\left(y_{1}, y_{2}\right) \Longleftrightarrow x_{1} \oplus y_{2}=x_{2} \oplus y_{1} .
$$

$\nabla$ is reflexive and symmetric, but not transitive. Therefore, we introduce another relation $\mathcal{R}$ defined by

$$
\left(x_{1}, x_{2}\right) \mathcal{R}\left(y_{1}, y_{2}\right) \Longleftrightarrow \begin{cases}\left(x_{1}, x_{2}\right) \nabla\left(y_{1}, y_{2}\right), & \text { when } x_{1} \neq x_{2} \text { and } y_{1} \neq y_{2}, \\ \left(x_{1}, x_{2}\right)=\left(y_{1}, y_{2}\right), & \text { otherwise. }\end{cases}
$$

$\mathcal{R}$ is an equivalence relation compatible with the operations $\oplus, \otimes, \ominus,||_{\oplus}, \bullet$, and the relation $\nabla$. Thus, we can define the quotient structure

$$
u \mathbb{R}=\mathbb{R}_{\max }^{2} / \mathcal{R} .
$$

This is called the symmetrized max-plus algebra [1,2]. Usually this is denoted by $\mathbb{S}$, but we use $u \mathbb{R}$ to imply it is somehow a whole set of ultradiscrete real numbers. We will also introduce $\mathrm{u} \mathbb{Z}, \mathrm{u} \mathbb{C}$ later.

Proposition A.1. We have three kinds of equivalence classes:

$$
\begin{aligned}
& \overline{(x,-\infty)}=\left\{(x, t): t \in \mathbb{R}_{\max } \text { and } x>t\right\}, \\
& \overline{(-\infty, x)}=\left\{(t, x): t \in \mathbb{R}_{\max } \text { and } t<x\right\}, \\
& \overline{(x, x)}=\{(x, x)\} .
\end{aligned}
$$

$\mathbb{R}_{\max }$ is embedded into $u \mathbb{R}$ by $x \longmapsto \overline{(x,-\infty)}$. Define

$$
\ominus \mathbb{R}_{\max }=\left\{\overline{(-\infty, x)}: x \in \mathbb{R}_{\max }\right\}, \quad \mathbb{R}_{\max }^{\bullet}=\left\{\overline{(x, x)}: x \in \mathbb{R}_{\max }\right\} .
$$

Then $u \mathbb{R}$ has a decomposition

$$
u \mathbb{R}=\mathbb{R}_{\max } \cup \ominus \mathbb{R}_{\max } \cup \mathbb{R}_{\text {max }}^{\bullet},
$$

and $\overline{(-\infty,-\infty)}$ is the only element which belongs to any two of the three sets. Thus, we simply write $x$ for $\overline{(x,-\infty)}, \ominus x$ for $\overline{(-\infty, x)}$, and $x$ for $\overline{(x, x)}$.

Define sign function $\operatorname{sgn} x$ by

$$
\operatorname{sgn} x= \begin{cases}0, & x \in \mathbb{R} \\ 0^{\bullet}, & x \in \mathbb{R}_{\max }^{\bullet} \\ \ominus 0, & x \in \ominus \mathbb{R}\end{cases}
$$

$x \in u \mathbb{R}$ is said to be positive if $\operatorname{sgn} x=0$, negative if $\operatorname{sgn} x=\ominus 0$, and balanced if $\operatorname{sgn} x=0^{\bullet}$.

Define $u \mathbb{R}^{\vee}=\mathbb{R}_{\max } \cup \ominus \mathbb{R}_{\max } \cdot x \in u \mathbb{R}$ is said to be signed if $x \in u \mathbb{R}^{\vee}$.

Proposition A.2. Let $\mathrm{uR}^{\otimes}$ denote the whole set of invertible elements in $\mathrm{u} \mathbb{R}$. Then,

$$
u \mathbb{R}^{\otimes}=u \mathbb{R}^{\vee} \backslash\{-\infty\}=u \mathbb{R} \backslash \mathbb{R}_{\max }^{\bullet} .
$$

Define $u \mathbb{Z}, u \mathbb{Z}^{\vee} \subset u \mathbb{R}$ by

$$
\mathrm{u} \mathbb{Z}=\{-\infty\} \cup \mathbb{Z} \cup \ominus \mathbb{Z} \cup \mathbb{Z}^{\bullet}, \quad \mathrm{u} \mathbb{Z}^{\vee}=\mathrm{u} \mathbb{Z} \cap u \mathbb{R}^{\vee}
$$

with obvious notations. $u \mathbb{Z}$ is a subdioid of $u \mathbb{R}$ and can be regarded as a whole set of ultradiscrete integers. $x \in \mathrm{u} \mathbb{Z}$ is said to be even if $|x|_{\oplus}$ is even, odd if $|x|_{\oplus}$ is odd. We do not define whether $-\infty$ is even or odd. We have of course

$$
\mathrm{u} \mathbb{Z}^{\otimes}=\mathrm{u} \mathbb{Z}^{\vee} \backslash\{-\infty\} .
$$




\section{A.1.3 Properties of balance relation}

We make much use of $\nabla$, rather than $\mathcal{R}$, since members of $\mathbb{R}_{\max }^{\bullet}$ can be regarded as a kind of null elements by virtue of the following proposition.

Proposition A.3. For any $x \in \mathrm{u} \mathbb{R}$,

$$
x \nabla-\infty \Longleftrightarrow x \in \mathbb{R}_{\max }^{\bullet} .
$$

Proposition A.4. For any $x \in u \mathbb{R}$ and $t \in \mathbb{R}_{\max }$,

$x \nabla t^{\bullet}$ and $x \notin \mathbb{R}_{\max }^{\bullet} \Longleftrightarrow|x|_{\oplus} \leq t$.

Proposition A.5. For any $x, y \in u \mathbb{R}$, we have

$x \nabla y \Longleftrightarrow x \ominus y \nabla-\infty$.

Proposition A.6. For any $x, y, z, w \in u \mathbb{R}$, we have

$x \nabla y$ and $z \nabla w \Longrightarrow x \oplus z \nabla y \oplus w$,

$x \nabla y \Longrightarrow x z \nabla y z$.

Proposition A.7 (weak substitution).

$x \nabla y, c y \nabla z$, and $y \in u \mathbb{R}^{\vee} \Longrightarrow c x \nabla z$.

Corollary A.8 (weak transitivity).

$x \nabla y, y \nabla z$, and $y \in u \mathbb{R}^{\vee} \Longrightarrow x \nabla z$.

Proposition A.9 (reduction of balances).

$x \nabla y$ and $x, y \in u \mathbb{R}^{\vee} \Longrightarrow x=y$.

\section{A.1.4 Matrices and determinants}

Let $\operatorname{uMat}(N, \mathrm{uR})$ denote the whole set of $N \times N$ matrices over $u \mathbb{R}$. Define addition $\oplus$ by

$$
\left(a_{i j}\right) \oplus\left(b_{i j}\right)=\left(a_{i j} \oplus b_{i j}\right)
$$

and multiplication $\otimes$ by

$$
\left(a_{i j}\right) \otimes\left(b_{i j}\right)=\left(c_{i j}\right), \quad c_{i j}=\bigoplus_{k} a_{i k} \otimes b_{k j}
$$

for any $\left(a_{i j}\right),\left(b_{i j}\right) \in \mathrm{uMat}(N, \mathrm{u} \mathbb{R})$. Then $\mathrm{uMat}(N, \mathrm{u} \mathbb{R})$ becomes a dioid, noncommutative when $N>1 . \ominus, \bullet$, and $\nabla$ are of course defined by

$$
\begin{aligned}
& \ominus\left(a_{i j}\right)=\left(\ominus a_{i j}\right), \quad\left(a_{i j}\right)^{\bullet}=\left(a_{i j}^{\bullet}\right), \\
& \left(a_{i j}\right) \nabla\left(b_{i j}\right) \Longleftrightarrow a_{i j} \nabla b_{i j} \text { for any } i, j
\end{aligned}
$$

respectively. $\left(a_{i j}\right) \in \operatorname{uMat}(N, \mathrm{u} \mathbb{R})$ is said to be signed if all the elements are signed. The whole set of signed elements in $\operatorname{uMat}(N, u \mathbb{R})$ is denoted by $u \operatorname{Mat}(N, u \mathbb{R})^{\vee}$. uR is embedded into $\operatorname{uMat}(N, \mathrm{uR})$ by

$$
x \longmapsto\left(\begin{array}{cccc}
x & -\infty & \cdots & -\infty \\
-\infty & x & \ddots & \vdots \\
\vdots & \ddots & \ddots & -\infty \\
-\infty & \cdots & -\infty & x
\end{array}\right) .
$$


For any permutation $\sigma \in S_{N}$, define $\operatorname{sgn}(\sigma)$ by

$$
\operatorname{sgn}(\sigma)= \begin{cases}0, & \text { when } \sigma \text { is even } \\ \ominus 0, & \text { when } \sigma \text { is odd. }\end{cases}
$$

And define the determinant of a matrix $A=\left(a_{i j}\right) \in \mathrm{uMat}(N, \mathrm{u} \mathbb{R})$ by

$$
\operatorname{det} A=\bigoplus_{\sigma} \operatorname{sgn}(\sigma) \bigotimes_{i} a_{i \sigma(i)}
$$

$\operatorname{det} A$ is also denoted by $|A|$ or $\left|a_{i j}\right|$.

Proposition A.10.

$$
\left|{ }^{t} A\right|=|A|
$$

where ${ }^{t} A$ denotes transposition of $A$.

\section{Proposition A.11.}

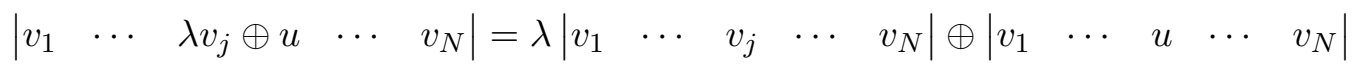

where $v_{j}={ }^{t}\left(a_{1 j}, \ldots, a_{N j}\right)$ and $u={ }^{t}\left(u_{1}, \ldots, u_{N}\right)$.

Proposition A.12. For any permutation $\sigma \in S_{N}$,

$$
\left|a_{i \sigma(j)}\right|=\operatorname{sgn}(\sigma)\left|a_{i j}\right|
$$

Corollary A.13. If $v_{j}=v_{k}$ for some $j \neq k$, then

$$
\left|\begin{array}{lll}
v_{1} & \cdots & v_{N}
\end{array}\right| \nabla-\infty
$$

Let $\operatorname{cof}_{i j}(A)$ denote the cofactor of $a_{i j}$ in $|A|$, which by definition satisfies

$$
|A|=\bigoplus_{i} a_{i j} \otimes \operatorname{cof}_{i j}(A)
$$

for any $j$. Define the adjacent matrix of $A$ by

$$
\operatorname{adj} A=\left(b_{i j}\right), \quad b_{i j}=\operatorname{cof}_{j i}(A) .
$$

\section{Theorem A.14.}

$$
\begin{aligned}
& \quad A \otimes \operatorname{adj} A \nabla|A|, \quad \operatorname{adj} A \otimes A \nabla|A| . \\
& \text { If }|A| \in \mathrm{u} \mathbb{R}^{\otimes} \text {, define } A^{-1} \text { by } \\
& A^{-1}=|A|^{-1} \text { adj } A .
\end{aligned}
$$

This is not a multiplicative inverse in general, but plays a similar role with regard to $\nabla$. Therefore we use the notation $A^{-1}$. 


\section{A.1.5 Ultradiscrete complex numbers}

It is well known that we can construct complex numbers by $2 \times 2$ real matrices, using

$$
i=\left(\begin{array}{cc}
0 & -1 \\
1 & 0
\end{array}\right)
$$

as the imaginary unit. Here we try to construct ultradiscrete complex numbers in a similar way.

Let $\mathrm{uMat}(N, \mathrm{u} \mathbb{R})$ denote the algebra of $N \times N$ matrices whose elements are in $u \mathbb{R}$. Define $I \in \mathrm{uMat}(2, \mathrm{u} \mathbb{R})$ by

$$
I=\left(\begin{array}{cc}
-\infty & \ominus 0 \\
0 & -\infty
\end{array}\right)
$$

We have

$$
I^{2}=\left(\begin{array}{cc}
-\infty & \ominus 0 \\
0 & -\infty
\end{array}\right)\left(\begin{array}{cc}
-\infty & \ominus 0 \\
0 & -\infty
\end{array}\right)=\left(\begin{array}{cc}
\ominus 0 & -\infty \\
-\infty & \ominus 0
\end{array}\right)=\ominus 0 .
$$

Define $\mathrm{u} \mathbb{C} \subset \operatorname{uMat}(2, \mathrm{u} \mathbb{R})$ by

$$
\mathrm{u} \mathbb{C}=\{x \oplus y I \mid x, y \in u \mathbb{R}\} .
$$

Proposition A.15. $\mathrm{u} \mathbb{C}$ is a commutative subdioid of $\mathrm{uMat}(2, \mathrm{u} \mathbb{R})$.

Proof. Obviously $\mathrm{u} \mathbb{C}$ includes $-\infty$ and 0 . For any $a \oplus b I, c \oplus d I \in \mathrm{u} \mathbb{C}$, we have

$$
\begin{aligned}
& (a \oplus b I) \oplus(c \oplus d I)=(a \oplus c) \oplus(b \oplus d) I \in \mathrm{u} \mathbb{C}, \\
& (a \oplus b I) \otimes(c \oplus d I)=(a c \ominus b d) \oplus(a d \oplus b c) I \in \mathrm{u} \mathbb{C} .
\end{aligned}
$$

And $\mathrm{u} \mathbb{C}$ is commutative because $I^{0}$ and $I^{1}$ are commutative.

When $z \in \mathrm{u} \mathbb{C}$ is expressed as $z=x+y I$ where $x, y \in u \mathbb{R}$, we write

$$
\mathrm{uRe} z=x, \quad \mathrm{uIm}=y .
$$

The whole set of signed elements of $\mathrm{u} \mathbb{C}$ is denoted by $u \mathbb{C}^{\vee}$.

If $\operatorname{det}(x \oplus y I)=x^{2} \oplus y^{2} \in \mathrm{u} \mathbb{R}^{\otimes}$, we have

$$
(x \oplus y I)^{-1}=\frac{x \ominus y I}{x^{2} \oplus y^{2}}
$$

and

$$
(x \oplus y I)(x \oplus y I)^{-1}=0 \oplus \frac{(x y)^{\bullet}}{x^{2} \oplus y^{2}} I \nabla 0 .
$$

\section{A.2 Ultradiscretization with negative numbers}

Ultradiscretization with negative numbers is presented in De Schutter et al. [4]. Here we reformulate it in a similar, but more convenient form for our purpose.

Let $f(s)$ and $g(s)$ be real functions. We say $f(s)$ is asymptotically equivalent to $g(s)$ if there exists a real number $s_{0}$ such that $g(s) \neq 0$ for any $s>s_{0}$ and

$$
\lim _{s \rightarrow \infty} \frac{f(s)}{g(s)}=1 \text {. }
$$


We also say $f(s)$ is asymptotically equivalent to 0 if there exists a real number $s_{1}$ such that $f(s)=0$ for any $s>s_{1}$. Asymptotic equivalence is an equivalence relation and denoted by $f(s) \sim g(s)$.

We are interested in asymptotic equivalence to exponential functions. If

$$
f(s) \sim \mu_{F} e^{\widetilde{F} s}, \quad \mu_{F} \in \mathbb{R}^{\times}, \quad \widetilde{F} \in \mathbb{R},
$$

we write

$$
f(s) \stackrel{\mathrm{ud}}{\longrightarrow} F, \quad F=S\left(\mu_{F}\right) \otimes \widetilde{F} \in u \mathbb{R}^{\vee},
$$

where

$$
S(\mu)= \begin{cases}0, & \mu>0 \\ \ominus 0, & \mu<0 .\end{cases}
$$

We regard $0 \sim \mu e^{(-\infty) s}$ for some $\mu \in \mathbb{R}^{\times}$and $0 \stackrel{\mathrm{ud}}{\longrightarrow}-\infty$ as a convention.

It is very important here to notice that $\mu_{F}$ is not restricted to positive numbers, unlike the usual ultradiscretization procedure.

Proposition A.16 (ultradiscretization of addition). Let $f(s), g_{1}(s), \ldots, g_{n}(s)$ be real functions satisfying

$$
f(s)=\sum_{k=1}^{n} g_{k}(s)
$$

and

$$
f(s) \stackrel{\mathrm{ud}}{\longrightarrow} F, \quad g_{k}(s) \stackrel{\mathrm{ud}}{\longrightarrow} G_{k} .
$$

Then,

$$
F \nabla \bigoplus_{k=1}^{n} G_{k} .
$$

Remark A.17. $f(s) \stackrel{\text { ud }}{\longrightarrow} F$ and $g(s) \stackrel{\text { ud }}{\longrightarrow} G$ do not imply $f(s)+g(s) \stackrel{\text { ud }}{\longrightarrow} F \oplus G$ because $f(s)+g(s)$ might be no longer asymptotically equivalent to exponential functions. But if $f(s)$, $g(s)$ can be expressed by power series in $\delta=\mu_{D} e^{\widetilde{D} s}$ where $\widetilde{D}<0$, this is not a problem because $f(s)+g(s)$ can also be expressed by a power series in $\delta$.

Proposition A.18 (ultradiscretization of multiplication). Let $f(s), g(s), h(s)$ be real functions satisfying

$$
f(s)=g(s) h(s)
$$

and

$$
g(s) \stackrel{\mathrm{ud}}{\longrightarrow} G, \quad h(s) \stackrel{\mathrm{ud}}{\longrightarrow} H .
$$

Then,

$$
f(s) \stackrel{\mathrm{ud}}{\longrightarrow} F=G \otimes H .
$$


Corollary A.19 (ultradiscretization of polynomials). Let real functions $f(s), g_{k l}(s)$ satisfy

$$
f(s)=\sum_{k=1}^{n} \prod_{l=1}^{m_{k}} g_{k l}(s)
$$

and

$$
f(s) \stackrel{\mathrm{ud}}{\longrightarrow} F, \quad g_{k l}(s) \stackrel{\mathrm{ud}}{\longrightarrow} G_{k l} .
$$

Then,

$$
F \nabla \bigoplus_{k=1}^{n} \bigotimes_{l=1}^{m_{k}} G_{k l} .
$$

\section{A.3 Ultradiscretization of matrices and complex numbers}

We also reformulate ultradiscretization of matrices in [4]. Extension to complex numbers is straightforward.

Consider a matrix-valued function $f(s)=\left(f_{i j}(s)\right): \mathbb{R} \rightarrow \operatorname{Mat}(N, \mathbb{R})$. If

$$
f_{i j}(s) \stackrel{\mathrm{ud}}{\longrightarrow} F_{i j},
$$

we write

$$
f(s) \stackrel{\mathrm{ud}}{\longrightarrow} F=\left(F_{i j}\right) \in \operatorname{uMat}(N, \mathrm{u} \mathbb{R}) .
$$

This is a componentwise property; there is no exponential functions of matrices.

Proposition A.20. Let matrix-valued functions $f(s), g_{k l}(s)$ satisfy

$$
f(s)=\sum_{k=1}^{n} \prod_{l=1}^{m_{k}} g_{k l}(s)
$$

and

$$
f(s) \stackrel{\mathrm{ud}}{\longrightarrow} F, \quad g_{k l}(s) \stackrel{\mathrm{ud}}{\longrightarrow} G_{k l} .
$$

Then,

$$
F \nabla \bigoplus_{k=1}^{n} \bigotimes_{l=1}^{m_{k}} G_{k l} .
$$

Considering $2 \times 2$-matrix construction of complex numbers, we have

$$
i=\left(\begin{array}{cc}
0 & -1 \\
1 & 0
\end{array}\right) \stackrel{\mathrm{ud}}{\longrightarrow} I=\left(\begin{array}{cc}
-\infty & \ominus 0 \\
0 & -\infty
\end{array}\right)
$$

Let $f(s)=u(s)+v(s) i$ where $u(s), v(s)$ are real functions. If

$$
u(s) \stackrel{\mathrm{ud}}{\longrightarrow} U, \quad v(s) \stackrel{\mathrm{ud}}{\longrightarrow} V,
$$

we have of course

$$
f(s) \stackrel{\mathrm{ud}}{\longrightarrow} F=U \oplus V I \in \mathrm{u} \mathbb{C} .
$$


Proposition A.21. Let complex-valued functions $f(s), g_{k l}(s)$ satisfy

$$
f(s)=\sum_{k=1}^{n} \prod_{l=1}^{m_{k}} g_{k l}(s)
$$

and

$$
f(s) \stackrel{\mathrm{ud}}{\longrightarrow} F, \quad g_{k l}(s) \stackrel{\mathrm{ud}}{\longrightarrow} G_{k l} .
$$

Then,

$$
F \nabla \bigoplus_{k=1}^{n} \bigotimes_{l=1}^{m_{k}} G_{k l}
$$

\section{B Quasideterminants}

Quasideterminants [5] are noncommutative extension of determinants, or, more precisely, determinants divided by cofactors. Here we describe the definition and some properties required for Theorem 3.7. See [5] for more detail.

Let $R$ be a ring and $\operatorname{Mat}(N, R)$ be the whole set of $N \times N$ matrices over $R . R$ is not commutative in general. For any $\left(a_{i j}\right),\left(b_{i j}\right) \in \operatorname{Mat}(N, R)$, define addition by

$$
\left(a_{i j}\right)+\left(b_{i j}\right)=\left(a_{i j}+b_{i j}\right)
$$

and multiplication by

$$
\left(a_{i j}\right)\left(b_{i j}\right)=\left(c_{i j}\right), \quad c_{i j}=\sum_{k=1}^{N} a_{i k} b_{k j} .
$$

Ordering of multiplication is important here.

For any $A=\left(a_{i j}\right) \in \operatorname{Mat}(N, R)$, define the $(p, q)$-th quasideterminant $|A|_{p q}$ by

$$
|A|_{p q}=a_{p q}-r_{p}^{q}\left(A^{p q}\right)^{-1} c_{q}^{p},
$$

where $r_{p}^{q}$ is the $p$-th row of $A$ without the $q$-th element, $c_{q}^{p}$ is the $q$-th column of $A$ without the $p$-th element, and $A^{p q}$ is $A$ without the $p$-th row and the $q$-th column. $|A|_{p q}$ is also written as

$$
|A|_{p q}=\left|\begin{array}{ccc}
a_{11} & \cdots & a_{1 N} \\
\vdots & a_{p q} & \vdots \\
a_{N 1} & \cdots & a_{N N}
\end{array}\right| .
$$

For example, we have

$$
\left|\begin{array}{ll}
a_{11} & a_{12} \\
a_{21} & a_{22}
\end{array}\right|=a_{11}-a_{12} a_{22}^{-1} a_{21}, \quad\left|\begin{array}{ll}
a_{11} & a_{12} \\
a_{21} & a_{22}
\end{array}\right|=a_{12}-a_{11} a_{21}^{-1} a_{22}
$$

and

$$
\begin{aligned}
\left|\begin{array}{lll}
a_{11} & a_{12} & a_{13} \\
a_{21} & a_{22} & a_{23} \\
a_{31} & a_{32} & a_{33}
\end{array}\right|= & a_{11}-\left(\begin{array}{ll}
a_{12} & a_{13}
\end{array}\right)\left(\begin{array}{cc}
a_{22} & a_{23} \\
a_{32} & a_{33}
\end{array}\right)^{-1}\left(\begin{array}{c}
a_{21} \\
a_{31}
\end{array}\right) \\
= & a_{11}-a_{12}\left(a_{22}-a_{23} a_{33}^{-1} a_{32}\right)^{-1} a_{21}-a_{13}\left(a_{23}-a_{22} a_{32}^{-1} a_{33}\right)^{-1} a_{21} \\
& -a_{12}\left(a_{32}-a_{33} a_{23}^{-1} a_{22}\right)^{-1} a_{31}-a_{13}\left(a_{33}-a_{32} a_{22}^{-1} a_{23}\right)^{-1} a_{31} .
\end{aligned}
$$


Proposition B.1. If we write $A^{-1}=\left(b_{i j}\right)$, we have

$$
b_{i j}=|A|_{j i}^{-1} .
$$

Proposition B.2. Quasideterminants are invariant under row and column permutations. (If the row or column contains the box, it is moved together.)

Proposition B.3 (homological relations). For $p_{1} \neq p_{2}, q_{1} \neq q_{2}, i \neq p, j \neq q$, we have the row homological relation

$$
|A|_{p q_{1}}\left|A^{p q_{2}}\right|_{i q_{1}}^{-1}+|A|_{p q_{2}}\left|A^{p q_{1}}\right|_{i q_{2}}^{-1}=0
$$

and the column homological relation

$$
\left|A^{p_{2} q}\right|_{p_{1} j}^{-1}|A|_{p_{1} q}+\left|A^{p_{1} q}\right|_{p_{2} j}^{-1}|A|_{p_{2} q}=0 .
$$

Proposition B.4 (Sylvester's identity). For any $A=\left(a_{i j}\right) \in \operatorname{Mat}(N, R)$, define $(N-k) \times(N-k)$ matrix $A_{0}$ by

$$
A_{0}=\left(a_{i j}\right), \quad k+1 \leq i, j \leq N
$$

and $k \times k$ matrix $C$ by

$$
C=\left(c_{i j}\right), \quad c_{i j}=\left|\begin{array}{cccc}
a_{i j} & a_{i(k+1)} & \cdots & a_{i N} \\
a_{(k+1) j} & & & \\
\vdots & & A_{0} & \\
a_{N j} & &
\end{array}\right| .
$$

Then

$$
|A|_{p q}=|C|_{p q} .
$$

\section{Acknowledgements}

The author would like to express his gratitude to Professor Tetsuji Tokihiro, who provided precise advices with a fine prospect. The author is also grateful to Professor Ralph Willox, who provided helpful comments for refining the results. In addition, the author thanks the anonymous referees for carefully reading the paper and giving many suggestions.

\section{References}

[1] Akian M., Cohen G., Gaubert S., Nikoukhah R., Quadrat J.P., Linear systems in (max,+) algebra, in Proceedings of the 29th IEEE Conference on Decision and Control (December, 1990, Honolulu, Hawaii), IEEE, 1990, 151-156.

[2] Baccelli F.L., Cohen G., Olsder G.J., Quadrat J.P., Synchronization and linearity. An algebra for discrete event systems, John Wiley \& Sons Ltd., Chichester, 1992, available at https://www.rocq.inria.fr/ metalau/cohen/SED/book-online.html.

[3] Date E., Jimbo M., Miwa T., Method for generating discrete soliton equations. III, J. Phys. Soc. Japan 52 (1983), 388-393.

[4] De Schutter B., De Moor B., The QR decomposition and the singular value decomposition in the symmetrized max-plus algebra revisited, SIAM Rev. 44 (2002), 417-454.

[5] Gelfand I., Gelfand S., Retakh V., Wilson R.L., Quasideterminants, Adv. Math. 193 (2005), 56-141.

[6] Grimshaw R., El G., Khusnutdinova K., Sine-Gordon equation, available at http://homepages.Iboro.ac. $\mathrm{uk} / \sim \operatorname{makk} / \mathrm{NLW}-\mathrm{L} 10 . \mathrm{pdf}$. 
[7] Hirota R., Nonlinear partial difference equations. III. Discrete sine-Gordon equation, J. Phys. Soc. Japan 43 (1977), 2079-2086.

[8] Isojima S., Grammaticos B., Ramani A., Satsuma J., Ultradiscretization without positivity, J. Phys. A: Math. Gen. 39 (2006), 3663-3672.

[9] Isojima S., Murata M., Nobe A., Satsuma J., An ultradiscretization of the sine-Gordon equation, Phys. Lett. A 331 (2004), 378-386.

[10] Isojima S., Satsuma J., On oscillatory solutions of the ultradiscrete sine-Gordon equation, JSIAM Lett. 1 (2009), 25-27.

[11] Kasman A., Lafortune S., When is negativity not a problem for the ultradiscrete limit?, J. Math. Phys. 47 (2006), 103510, 16 pages.

[12] Kondo K., Sato-theoretic construction of solutions to noncommutative integrable systems, Phys. Lett. A 375 (2011), 488-492.

[13] Lax P.D., Integrals of nonlinear equations of evolution and solitary waves, Comm. Pure Appl. Math. 21 (1968), 467-490.

[14] Lechtenfeld O., Mazzanti L., Penati S., Popov A.D., Tamassia L., Integrable noncommutative sine-Gordon model, Nuclear Phys. B 705 (2005), 477-503, hep-th/0406065.

[15] Mimura N., Isojima S., Murata M., Satsuma J., Singularity confinement test for ultradiscrete equations with parity variables, J. Phys. A: Math. Theor. 42 (2009), 315206, 7 pages.

[16] Nijhoff F., Capel H., The discrete Korteweg-de Vries equation, Acta Appl. Math. 39 (1995), 133-158.

[17] Nimmo J.J.C., On a non-abelian Hirota-Miwa equation, J. Phys. A: Math. Gen. 39 (2006), 5053-5065.

[18] Ochiai T., Nacher J.C., Inversible max-plus algebras and integrable systems, J. Math. Phys. 46 (2005), 063507, 17 pages, nlin.SI/0405067.

[19] Ormerod C.M., Hypergeometric solutions to an ultradiscrete Painlevé equation, J. Nonlinear Math. Phys. 17 (2010), 87-102, nlin.SI/0610048.

[20] Quispel G.R.W., Capel H.W., Scully J., Piecewise-linear soliton equations and piecewise-linear integrable maps, J. Phys. A: Math. Gen. 34 (2001), 2491-2503.

[21] Takahashi D., Satsuma J., A soliton cellular automaton, J. Phys. Soc. Japan 59 (1990), 3514-3519.

[22] Tokihiro T., Takahashi D., Matsukidaira J., Satsuma J., From soliton equations to integrable cellular automata through a limiting procedure, Phys. Rev. Lett. 76 (1996), 3247-3250.

[23] Yajima T., Nakajima K., Asano N., Max-plus algebra for complex variables and its applications to discrete Fourier transformation and partial difference equations, J. Phys. Soc. Japan 75 (2006), 064001, 7 pages, nlin.SI/0505056. 